\title{
Distinct growth hormone receptor signaling modes regulate skeletal muscle development and insulin sensitivity in mice
}

\author{
Mahendra D. Mavalli, ${ }^{1}$ Douglas J. DiGirolamo, ${ }^{1}$ Yong Fan, ${ }^{2}$ Ryan C. Riddle, ${ }^{1}$ \\ Kenneth S. Campbell, ${ }^{3}$ Thomas van Groen, ${ }^{4}$ Stuart J. Frank, 4,5,6 Mark A. Sperling, ${ }^{2}$ \\ Karyn A. Esser, ${ }^{3}$ Marcas M. Bamman, ${ }^{7}$ and Thomas L. Clemens ${ }^{1,8}$ \\ 1Department of Orthopaedic Surgery, Johns Hopkins University School of Medicine, Baltimore, Maryland, USA. \\ 2Department of Pediatrics, University of Pittsburgh School of Medicine, Pittsburgh, Pennsylvania, USA. ${ }^{3}$ Center for Muscle Biology, \\ University of Kentucky College of Medicine, Lexington, Kentucky, USA. ${ }^{4}$ Department of Cell Biology and ${ }^{5}$ Department of Medicine, \\ Division of Endocrinology, Diabetes and Metabolism, University of Alabama at Birmingham, Birmingham, Alabama, USA. \\ ${ }^{6}$ Endocrinology Section, Medical Service, Veterans Affairs Medical Center, Birmingham, Alabama, USA. ${ }^{7}$ Department of Physiology and Biophysics, \\ University of Alabama at Birmingham, Birmingham, Alabama, USA. ${ }^{8}$ Veterans Administration Medical Center, Baltimore, Maryland, USA.
}

\begin{abstract}
Skeletal muscle development, nutrient uptake, and nutrient utilization is largely coordinated by growth hormone (GH) and its downstream effectors, in particular, IGF-1. However, it is not clear which effects of GH on skeletal muscle are direct and which are secondary to GH-induced IGF-1 expression. Thus, we generated mice lacking either GH receptor (GHR) or IGF-1 receptor (IGF-1R) specifically in skeletal muscle. Both exhibited impaired skeletal muscle development characterized by reductions in myofiber number and area as well as accompanying deficiencies in functional performance. Defective skeletal muscle development, in both GHR and IGF-1R mutants, was attributable to diminished myoblast fusion and associated with compromised nuclear factor of activated $T$ cells import and activity. Strikingly, mice lacking GHR developed metabolic features that were not observed in the IGF-1R mutants, including marked peripheral adiposity, insulin resistance, and glucose intolerance. Insulin resistance in GHR-deficient myotubes derived from reduced IR protein abundance and increased inhibitory phosphorylation of IRS-1 on Ser 1101. These results identify distinct signaling pathways through which GHR regulates skeletal muscle development and modulates nutrient metabolism.
\end{abstract}

\section{Introduction}

Mammalian skeletal muscle has evolved to perform a diverse set of functions, including locomotion, breathing, protecting internal organs, and coordinating global energy expenditure. Skeletal muscle is formed and regenerated through a highly regulated process characterized by myoblast differentiation and fusion into multinucleated syncytia. During embryonic development, specification of mesodermal precursor cells into the myogenic lineage is controlled by signals from surrounding tissues and requires upregulation of several factors, including paired-box transcription factor 7 (pax-7) and basic helix-loop-helix transcriptional activators of the myogenic regulatory factor family, MyoD and Myf-5 (1). The proliferating precursor cells/myoblasts withdraw from the cell cycle and initiate muscle-specific gene expression $(2,3)$. Myoblasts then initially fuse to form nascent myotubes, with relatively few nuclei, through a highly ordered set of cellular events, including recognition, adhesion, alignment, and membrane union. Subsequent recruitment and fusion of additional myoblasts gives rise to multinucleated myotubes that ultimately mature to give rise to skeletal muscle fibers. The fusion process is controlled, in part, by the actions of calcium-sensitive transcription factors of the nuclear factor of activated T cells (NFAT) family $(4,5)$. During myoblast fusion,

Authorship note: Mahendra D. Mavalli and Douglas J. DiGirolamo contributed equally to this work.

Conflict of interest: The authors have declared that no conflict of interest exists. Citation for this article: J Clin Invest. 2010;120(11):4007-4020. doi:10.1172/JCI42447. the nuclear translocation of NFATc2 transcriptionally activates IL-4, a cytokine essential for myoblast recruitment (6).

The growth hormone/IGF-1 (GH/IGF-1) axis represents an important physiological regulatory mechanism for coordinating postnatal skeletal muscle expansion and hypertrophy. Administration of GH to both animals and GH-deficient humans improves muscle strength and reduces body fat (7-9). Moreover, recent studies have shown that mice globally deficient in GH receptor (GHR) have reduced muscle mass with defective myofiber specification and growth (10). Such studies clearly demonstrate the importance of GH in skeletal muscle development but do not address the mechanisms responsible for these effects.

GH exerts growth-promoting and metabolic effects in target tissues (11) by binding to the transmembrane GHR and triggering enhanced GHR association with, and activation of, the cytoplasmic tyrosine kinase JAK2 $(12,13)$. Three major signaling systems activated in response to GH include STATs (most notably STAT5b), phosphoinositide $\underline{3}$-kinase (PI3K), and Erk $(14,15)$. STAT5b activation by GH results in transcriptional activation of GH target genes, including IGF-1 $(16,17)$. Many but not all of the anabolic effects of $\mathrm{GH}$ are exerted indirectly via this stimulation of IGF-1 from liver and peripheral tissues (18-27).

IGF-1 is a small polypeptide with homology to proinsulin, which is produced by many cell types. IGF- 1 signals via the type- 1 IGF-1 receptor (IGF-1R), a widely expressed cell surface heterotetramer, highly similar to the IR, which possesses intrinsic kinase activity in its cytoplasmic domains $(28,29)$. Activated IGF-1R engages the Erk and PI3K pathways via phosphorylation of SHC 
and IRS- 1 to induce proliferation, inhibit apoptosis, and elicit a number of other cellular responses $(30,31)$. The availability of either systemic or locally produced IGF-1 to its cognate receptor is determined by a multicomponent delivery system composed of a family of IGF-binding proteins (32-34), which serve to prevent or facilitate access to IGF-1R.

In this study, we sought to define the mechanisms of GH action in skeletal muscle and specifically attempted to distinguish direct mechanisms of $\mathrm{GH}$ action from those mediated indirectly by IGF-1. To accomplish this, we created genetic mouse models with targeted disruption of GHR or IGF-1R, specifically in skeletal muscle. Our studies using these mice show that GH mediates skeletal muscle development by enhancing myoblast fusion in an IGF-1-dependent manner. By contrast, disruption of the GHR in skeletal muscle produces marked alterations in muscle nutrient uptake and insulin sensitivity in an IGF-1-independent manner.

\section{Results}

GH-induced myoblast proliferation and fusion is mediated by IGF-1. The anabolic effects of GH in skeletal muscle are well documented (7-9), but despite some evidence to suggest that these effects might be mediated by IGF-1 (35), the precise mode of GH action in skeletal muscle remains unclear. To begin to define the mechanism of GH action in skeletal muscle in vitro, we examined the effect of GH on IGF-1 production, myoblast proliferation, and myoblast fusion, using a primary myoblast culture system that has been described previously (36) (Supplemental Figure 1, A-F; supplemental material available online with this article; doi:10.1172/JCI42447DS1). Treatment of primary myoblasts with GH acutely increased If 1 mRNA by 3 hours, with maximal mRNA levels achieved by 6 hours (Figure 1A). Further, acute exposure of myoblasts to GH did not affect proliferation, as indexed by BrdU incorporation at 8 hours (prior to GH-induced IGF-1 protein production), whereas exogenous IGF-1 significantly increased primary myoblast proliferation (Figure 1B). These results are compatible with a mechanism in which GH induces myoblast production of IGF-1, and this IGF-1, in turn, stimulates myoblast proliferation through an autocrine mechanism.

We next examined the effect of GH on myoblast fusion. In this in vitro system, myoblasts fuse to form multinucleated myotubes in 2 stages. In the first phase ( 24 hours after induction of differentiation), 2 myoblasts fuse to form a nascent myotube. During the next phase (hours 24-48), additional myoblasts fuse to this nascent myotube to increase the number of nuclei, ultimately producing a larger, fully differentiated myotube. Myoblasts exposed to GH during the first 24-hour period (nascent myotube formation) fused similarly to controls, as assessed by the number of nuclei per myotube (Figure 1C). By contrast, cultures treated with GH for the entire 48-hour period of differentiation, or alternatively only during hours 24-48 (myonuclei accumulation), had significantly higher nuclei/myotube ratios (Figure 1C). This result suggests that $\mathrm{GH}$ affects myofiber development by stimulating accumulation of additional myonuclei into nascent myotubes.

We next sought to determine the requirement of GHR for basal myoblast fusion. Primary myoblasts isolated from mice carrying homozygous floxed Ghr alleles were infected with adenoviruses expressing either Cre-recombinase (adenoCre) to selectively excise the GHR (Supplemental Figure 1G) or adenoGFP as a control. Disruption of GHR did not alter basal myoblast fusion (Figure 1D), suggesting that GHR is not essential for basal levels of fusion, at least under these experimental conditions in vitro. As expected, disruption of GHR abolished GH-stimulated fusion observed in control myoblasts (Figure 1D). Addition of IGF-1 to myoblasts significantly increased the number of nuclei per myotube, regardless of GHR status (Figure 1D). Moreover, primary myoblasts lacking the IGF-1R, generated in a similar fashion as those above (Supplemental Figure $1 \mathrm{H}$ ), failed to fuse in response to $\mathrm{GH}$ (Figure 1E). To ensure that deletion of IGF-1R did not introduce a critical defect, such that myoblasts would be unable to respond to any fusion stimulus, control and $\Delta \mathrm{IGF}-1 \mathrm{R}$ myoblasts were also treated with IL-4, which is known to increase myoblast fusion (4). IL-4 did not significantly increase the number of nuclei per myotube in $\triangle \mathrm{IGF}-1 \mathrm{R}$ myoblasts (Supplemental Figure 1I). However, IL-4 increased the percentage of fused myoblasts (fusion index), regardless of IGF-1R status (Supplemental Figure 1J). By contrast, GH had no effect on the number of nuclei per myotube or the fusion index in $\triangle \mathrm{IGF}-1 \mathrm{R}$ myoblasts (Supplemental Figure 1, I and J). Taken together, these results suggest that the anabolic effect of $\mathrm{GH}$ to increase myoblast fusion is mediated by IGF-1. Further, IGF-1R appears to be critical for the accrual of myonuclei into nascent myotubes, at least in the context of our in vitro model.

GH induces myoblast fusion via NFATc2-induced IL-4 production. As described above, previous studies have implicated the NFAT family of transcription factors as critical mediators of myoblast fusion $(4,5)$. To investigate the potential involvement of the NFAT/IL-4 pathway in GH-induced myoblast fusion, we determined the effects of GH and IGF-1 on NFAT abundance and nuclear import. Initial studies indicated that NFATc2 was abundantly expressed by primary mouse myoblasts, whereas other NFAT isoforms were either absent or expressed at low levels (data not shown). Treatment of primary myoblasts with GH or IGF-1 for 1 hour induced NFATc2 nuclear localization (Figure 1F). Moreover, myoblasts carrying an NFAT luciferase reporter that were treated with either GH or IGF-1 significantly increased reporter activity over that observed in untreated cells (Figure 1G). Additionally, GH increased Il4 mRNA expression in primary myoblasts (Figure $1 \mathrm{H}$ ), in accordance with previous studies (6). These findings suggest that GH and/or IGF-1 intersect with the NFATc2/IL-4 pathway to promote primary myoblast fusion.

GHR is required for normal myofiber specification, myonuclei accumulation, and muscle function. To examine the role of individual components of the GH/IGF-1 axis in skeletal muscle development in vivo, we created 2 mouse models that lacked either GHR or IGF-1R in skeletal muscle. Details about the generation of these animals are illustrated in Supplemental Figure 2 and described in the Methods. Mice lacking GHR ( $\Delta$ GHR mice) were born at the expected Mendelian frequency and were viable. Deletion of $G h r$ mRNA from skeletal muscle was confirmed by real-time PCR (Figure 2A), and importantly, removal of the GHR from skeletal muscle did not affect Igflr mRNA expression levels (Figure 2B). Expression of Igfl mRNA in skeletal muscle was increased at 6 weeks (Figure 2C), possibly a result of compensation by other pathways known to activate IGF-1 production in skeletal muscle, most likely androgens $(37,38)$. However, Igf1 mRNA in $\triangle$ GHR mutants declined to levels below those observed in control animals by 16 weeks (Figure 2C). Serum levels of GH and IGF-1 in $\Delta$ GHR mice, also measured at 16 weeks of age, were not different from control mice (data not shown). Quantitative histomorphometric analysis of the medial gastrocnemius muscle from $\triangle \mathrm{GHR}$ and control littermates was performed at 6 and 16 weeks (Figure 2, D-G). At 6 weeks, the number of myonuclei per 100 myofibers in cross-section was 
A

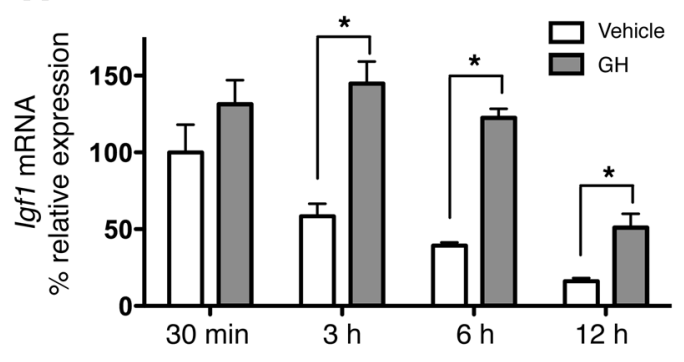

B

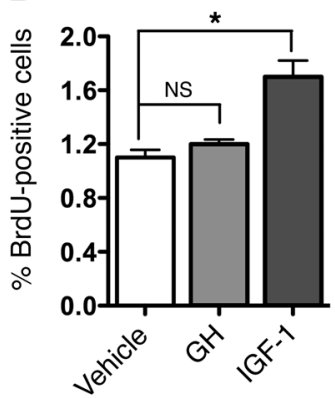

C $\square 0-48 \mathrm{~h} \square 0-24 \mathrm{~h} \square 24-48 \mathrm{~h}$

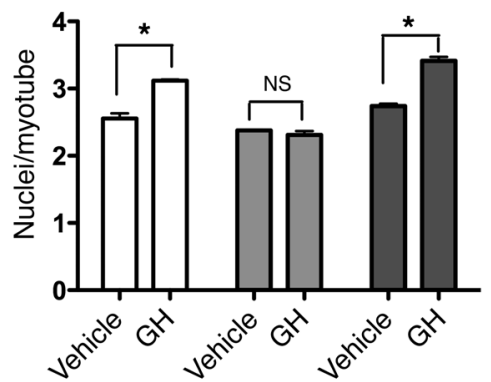

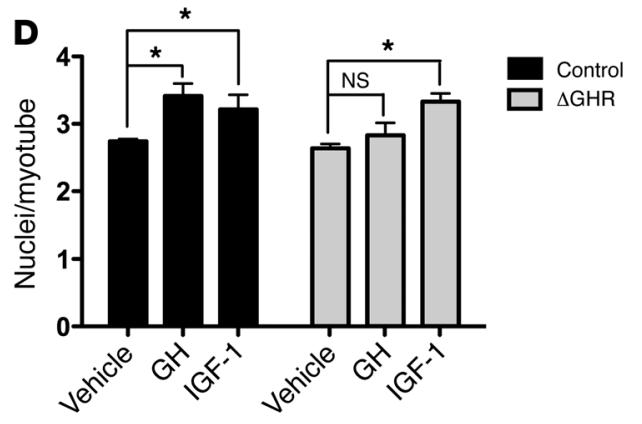

E

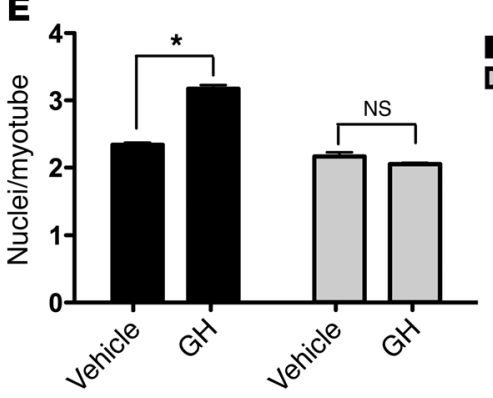

$\mathbf{F}$

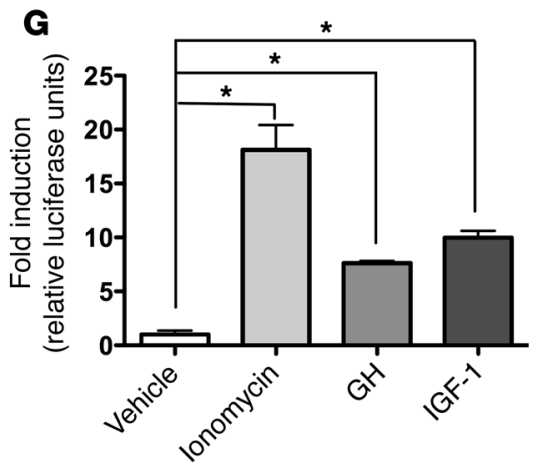

H

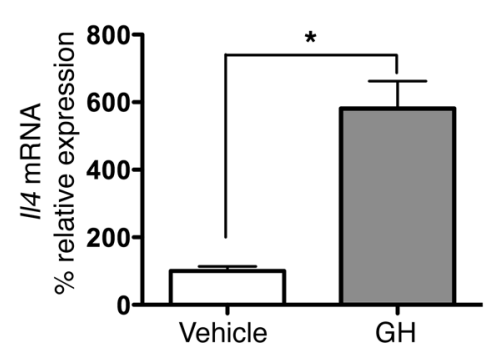

\section{Figure 1}

GH-induced myoblast proliferation and fusion are mediated by IGF-1 production and intersect the NFATc2/IL-4 pathway. (A) Primary mouse myoblasts were serum starved for 10 hours before stimulation with GH. Real-time PCR was performed using primers for IGF-1 at indicated times. (B) Myoblasts were serum starved for 10 hours before stimulation with GH or IGF-1 for 8 hours, with BrdU exposure for the full 8 hours of treatment. The percentage of BrdU-positive cells was assessed by flow cytometry. (C) Myoblasts were induced to differentiate and treated with GH for 0-48 hours, 0-24 hours, or 24-48 hours of differentiation. (D) GHR-floxed myoblasts were infected with adenoGFP (control) or adenoCre $(\Delta G H R)$ and differentiated in the presence of vehicle, GH, or IGF-1 for 48 hours. (E) IGF-1R-floxed myoblasts were infected with adenoGFP (control) or adenoCre $(\Delta \mathrm{IGF}-1 \mathrm{R})$ as treated in $\mathbf{D}$. (F) Wild-type myoblasts were serum starved for 10 hours before stimulation with vehicle (Veh), $\mathrm{GH}$, or IGF-1 for 1 hour and harvest of nuclear protein extracts. (G) Myoblasts were transfected with an NFAT luciferase reporter construct before induction of differentiation for 24 hours and treatment with vehicle, ionomycin, GH, or IGF-1 for 5 hours. (H) Myoblasts were serum starved for 10 hours before treatment with vehicle or GH for 5 hours. Real-time PCR was performed using primers for IL-4. All data shown is representative of at least 3 separate experiments performed from separate muscle cell preparations. Error bars indicate SEM. ${ }^{\star} P<0.05$.

lower in $\triangle \mathrm{GHR}$ mice compared with that of control littermates for both fiber types at both time points (Figure 2, $\mathrm{H}$ and $\mathrm{K}$ ). The proportion of type I fibers was lower in the $\Delta \mathrm{GHR}$ gastrocnemius compared with that of controls (Figure 2I), whereas the proportion of type II fibers were concomitantly higher than that of type I fibers (Figure 2I). At 6 weeks, type I and type II myofiber sizes did not differ between $\Delta \mathrm{GHR}$ and control mice (Figure 2J). However, $\triangle$ GHR mice failed to attain myofiber diameters comparable with those of controls for both type I and type II fibers by 16 weeks of age (Figure $2 \mathrm{M}$ ). These changes in muscle physiology persisted through 26 weeks of age (Supplemental Figure 3 ). The reduced number of myonuclei and smaller myofibers in $\Delta \mathrm{GHR}$ mice are consistent with deficient myoblast fusion demonstrated in vitro. Taken together, these results document the requirement of GHR for proper skeletal muscle development in vivo.

We next assessed the functional consequences of GHR disruption in skeletal muscle. Groups of 26-week-old control and $\Delta \mathrm{GHR}$ mice were subjected to the standard grip strength and rotarod tests. Mice were analyzed at 26 weeks of age, because, at this time, body weight reached a plateau in both $\triangle$ GHR and control mice (see below), and therefore, any developmental changes resulting from GHR deletion in skeletal muscle would be expected to have reached a steady state. At this time point, grip strength was lower (Figure $2 \mathrm{~N}$ ) and measures of rotarod endurance performance 
A
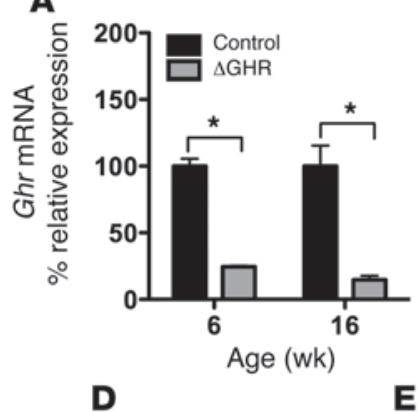

B

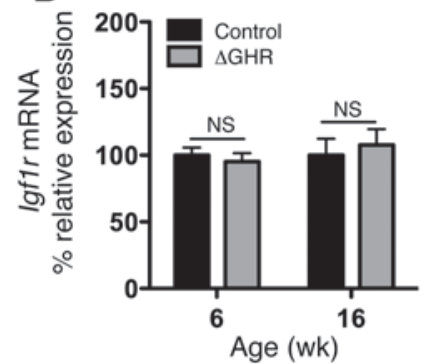

F

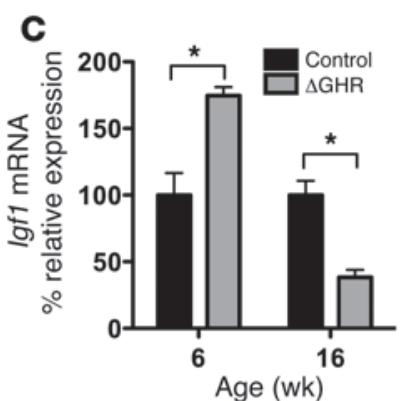

G

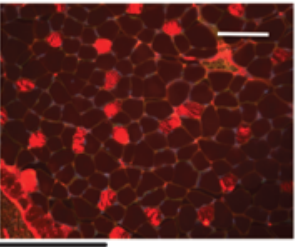

6 wk

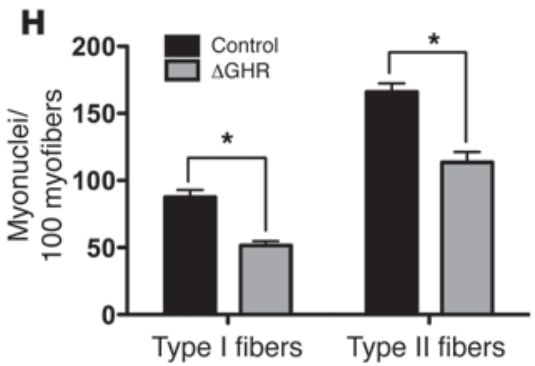

K
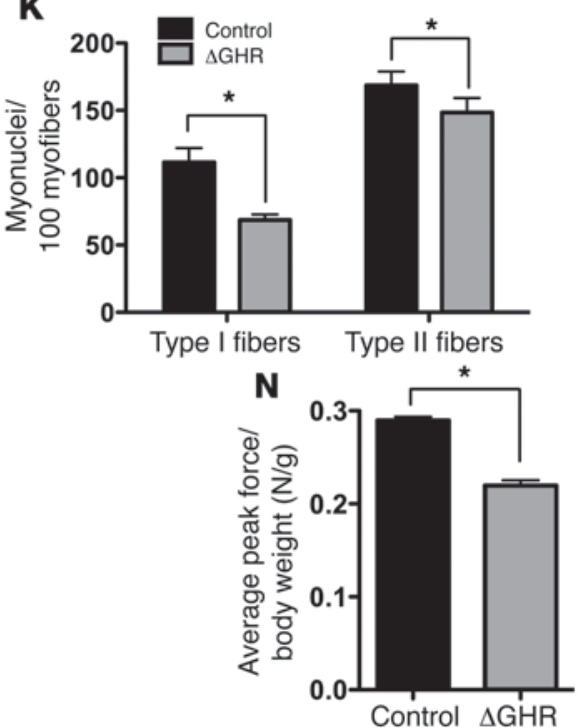

16 wk

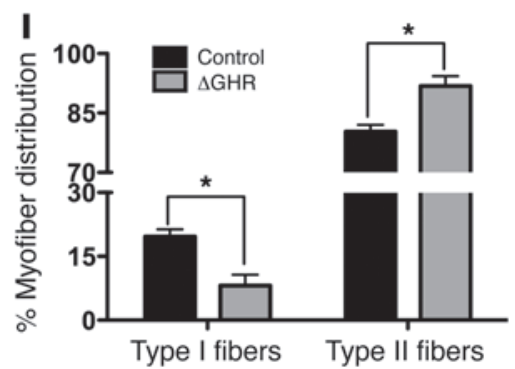

L

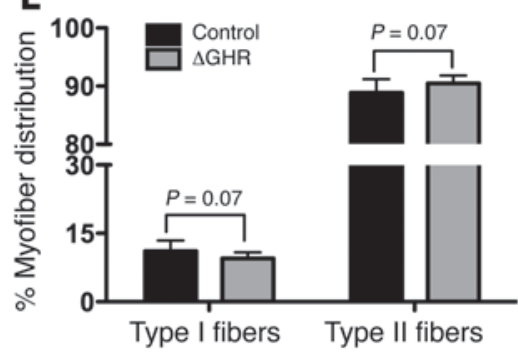

0

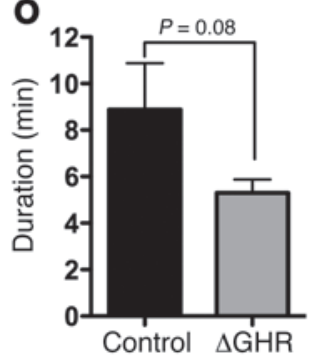

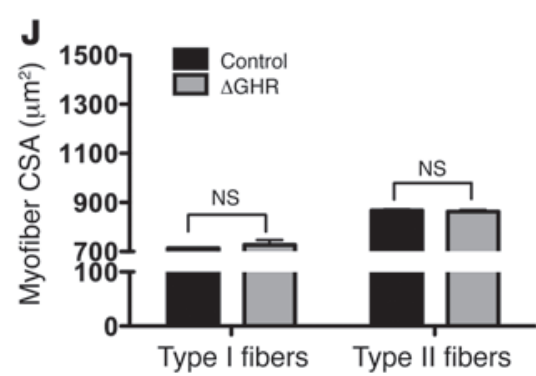
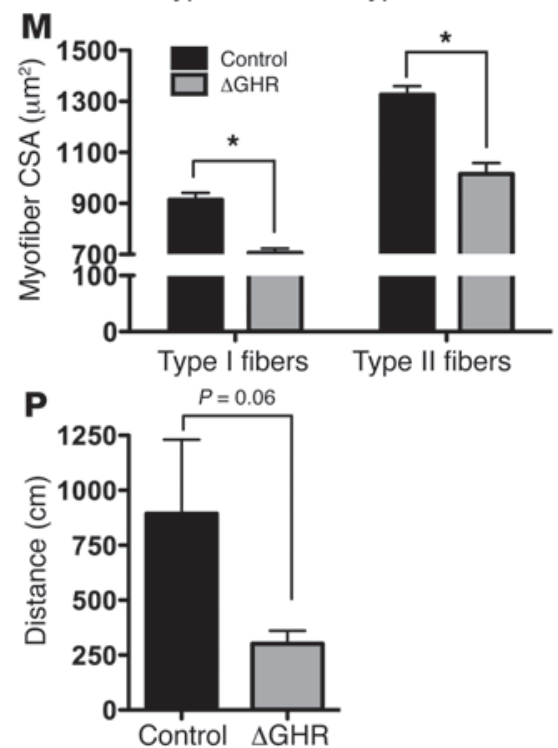

Figure 2

GHR is required for normal myofiber specification, myonuclei accumulation, and muscle function. mRNA was harvested from the gastrocnemius muscles of control and $\Delta$ GHR mice, and real-time PCR was performed using primers for (A) Ghr, (B) Igf1r, and (C) Igf1. (D-G) Sections of medial gastrocnemius from control and $\triangle \mathrm{GHR}$ mice were visualized using antibodies directed against MHC type I and laminin and counterstained with Hoechst 33258 to visualize myonuclei. Scale bars: $80 \mu \mathrm{m}$. (H-M) Histomorphometric analyses of myonuclei per 100 myofibers, percentage myofiber distribution, and myofiber diameter (CSA) were performed on sections described above for $(\mathbf{H}-\mathbf{J})$ 6-week-old and (K-M) 16-week-old control and $\Delta G H R$ mice. Muscle performance in 26-week-old control and $\Delta G H R$ mice was assessed by $(\mathbf{N})$ grip strength and $(\mathbf{O}$ and $\mathbf{P})$ rotarod testing, as described in Methods. For all studies shown, $n=6$ for control and $\Delta$ GHR at all time points. Error bars indicate SEM. ${ }^{\star} P<0.05$. 
A

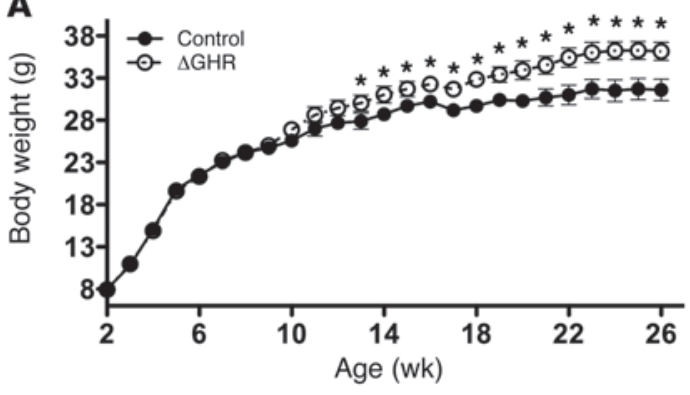

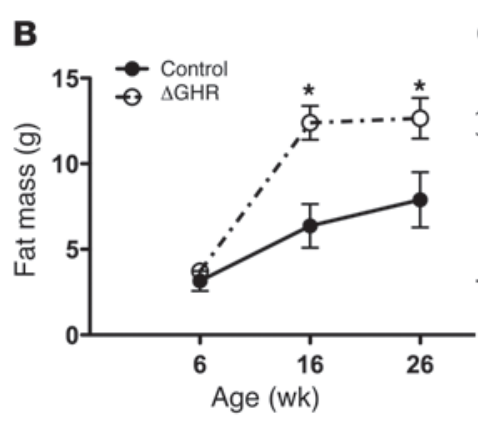

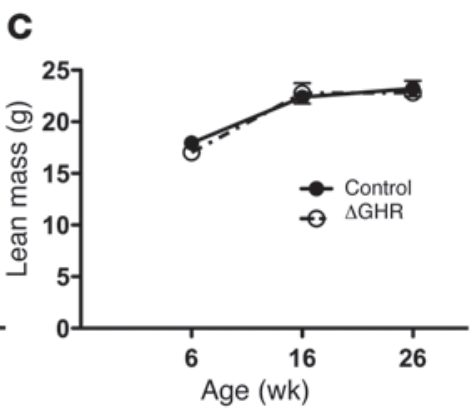

E

$\triangle \mathrm{GHR}$
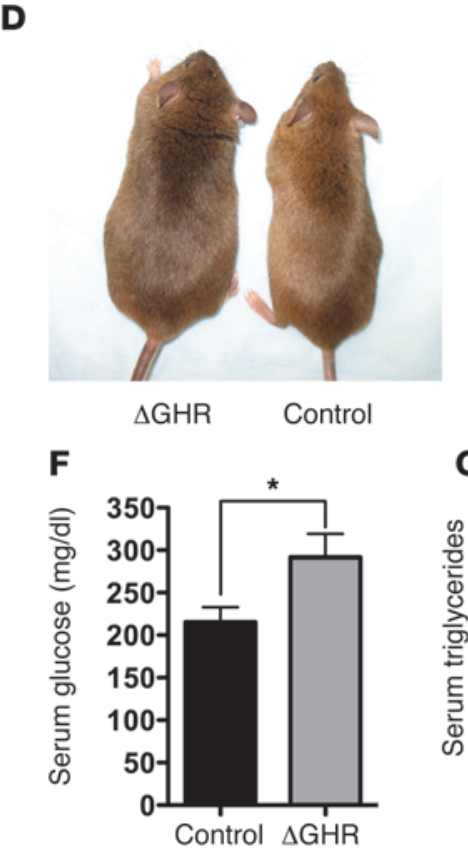

G

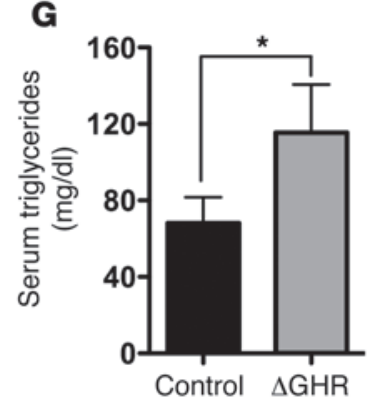

H

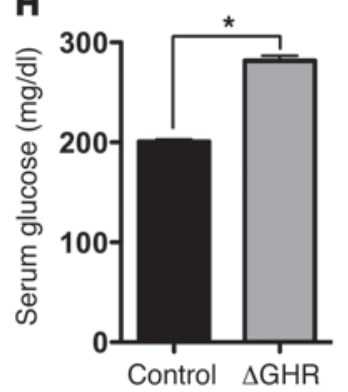

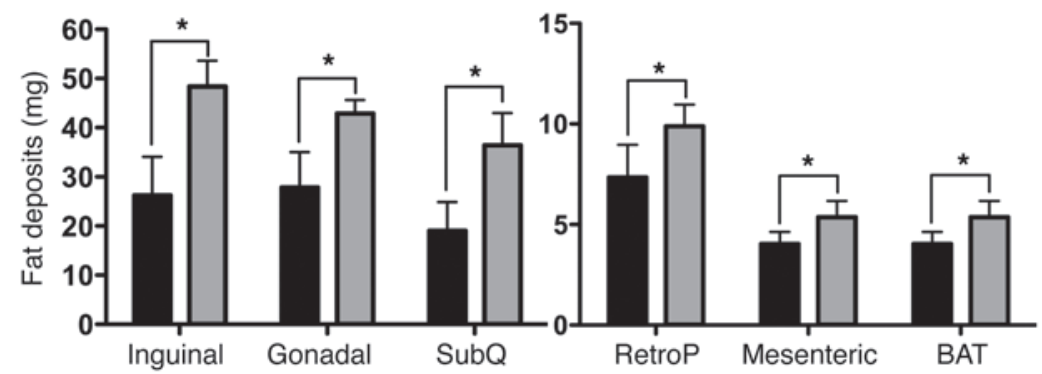
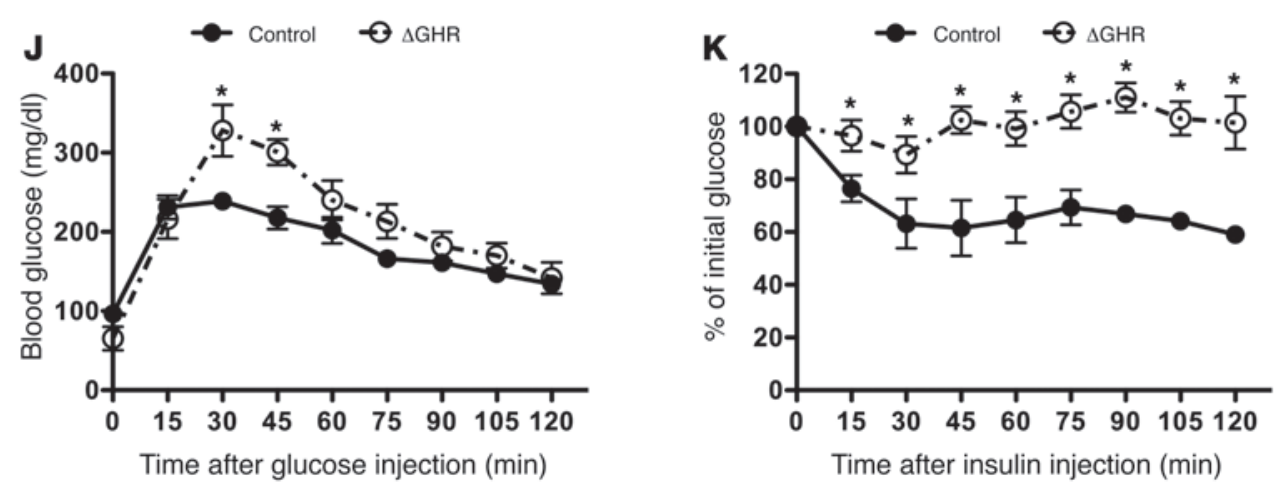

Figure 3

Loss of GHR in skeletal muscle causes peripheral adiposity, glucose intolerance, and insulin resistance. (A) Control $(n=9)$ and $\Delta$ GHR $(n=11)$ mice were weighed weekly from 2 to 26 weeks of age (image in $\mathbf{D}$ was taken at 26 weeks). qMR analysis of (B) fat and (C) lean mass in control and $\Delta$ GHR mice at 6,16 , and 26 weeks of age. (E) Fat pad mass at 26 weeks. SubQ, subcutaneous; RetroP, retroperitoneal; BAT, brown adipose tissue. ( $\mathbf{F}-\mathbf{I})$ Ad libitum serum glucose and triglyceride concentration at ( $\mathbf{F}$ and $\mathbf{G}) 16$ weeks and $(\mathbf{H}$ and $\mathbf{I}) 26$ weeks of age. Control and $\Delta$ GHR mice were also subjected to (J) GTTs and (K) ITTs, as described in Methods. Unless otherwise indicated, $n=6$ for control and $\Delta$ GHR at all time points. Error bars indicate SEM. ${ }^{*} P<0.05$.

tended to be lower (Figure 2, O and P) in $\triangle$ GHR mice compared with controls. These results suggest that the disruption of GHR in skeletal muscle and the consequent histomorphometric changes in myofiber type and size and myonuclei number result in functionally impaired skeletal muscle.
Loss of GHR in skeletal muscle causes peripheral adiposity, glucose intolerance, and insulin resistance. Sequential measurement of the body weights of $\triangle$ GHR mice showed an unanticipated increase in body weight of $\triangle$ GHR mutant mice relative to that of their control littermates (Figure 3, A and D) that first became apparent 

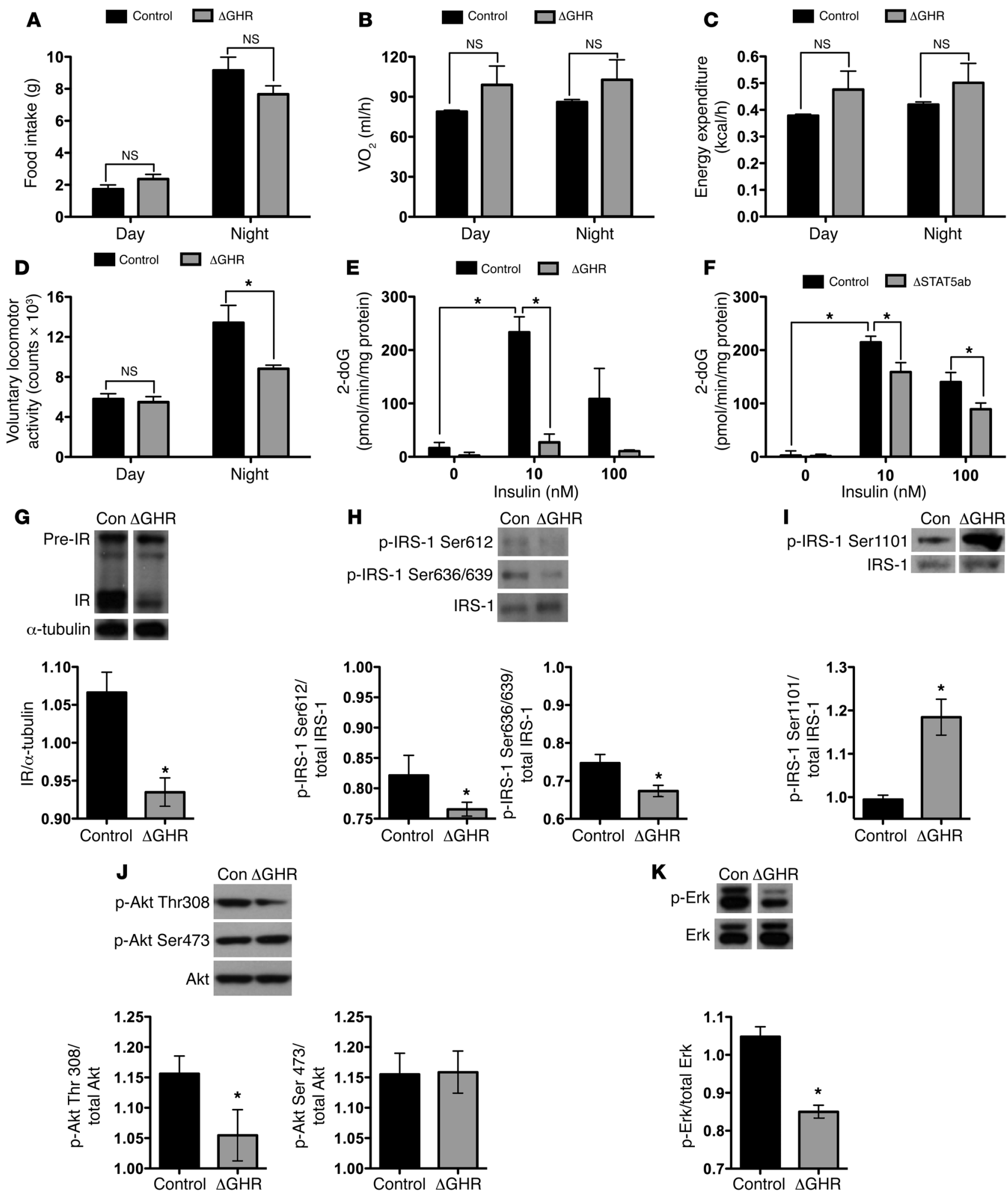


\section{Figure 4}

Metabolic phenotype of $\Delta \mathrm{GHR}$ mice is associated with skeletal muscle insulin resistance. (A) Food intake of 26-week-old control and $\Delta G H R$ mice. (B) Oxygen consumption $\left(\mathrm{VO}_{2}\right)$ and $(\mathbf{C})$ energy expenditure, as assessed by indirect calorimetry. (D) Voluntary locomotor activity assessed by computer-monitored infrared beam breaks ( $n=4$ control and $\triangle$ GHR mice for A-D). (E and F) Myoblasts carrying (E) floxed GHR alleles or (F) floxed STAT5ab alleles were infected with adenoGFP (control) or adenoCre ( $\triangle \mathrm{GHR}$ or $\triangle \mathrm{STAT5} 5 \mathrm{ab}$, respectively). Myoblasts were then serum starved for 12 hours, before a 1-hour pretreatment with vehicle or insulin, and then pulsed with 2-doG. Radiolabeled glucose uptake was determined by liquid scintillation counting. (G-K) Protein lysates were harvested from differentiated control and $\Delta G H R$ primary myotubes and subjected to SDS-PAGE analysis. Membranes were immunoblotted with antibodies directed against the proteins indicated. Membranes were stripped and reprobed for the indicated loading control protein. In some cases, additional irrelevant lanes between those shown were removed (indicated by separating white space). Bands for the indicated proteins were quantified based on gray density, as described in the Methods, and normalized against respective loading control proteins. All in vitro data shown is representative of at least 3 separate experiments performed from separate muscle cell preparations. Error bars indicate SEM. ${ }^{*} P<0.05$.

at 12 weeks. Quantitative magnetic resonance (qMR) showed that $\triangle$ GHR mice had increased fat mass (Figure $3 \mathrm{~B}$ ), compared with control littermates, with no significant differences in lean mass (Figure 3C). Fat pad mass was higher at all sites in $\triangle \mathrm{GHR}$ mice relative to that of controls (Figure 3E).

The peripheral adiposity observed in $\triangle$ GHR mice suggested disturbances in nutrient metabolism brought about by loss of $\mathrm{GH}$ action in skeletal muscle. Consistent with this idea, $\Delta \mathrm{GHR}$ mice had elevated serum glucose (Figure 3, F and $\mathrm{H}$ ) and triglyceride levels (Figure 3, G and I) in the fed state, although no differences were observed in serum insulin concentration (data not shown). These biochemical features, together with the changes in body composition in the $\Delta \mathrm{GHR}$ mice, indicated the development of insulin resistance. We therefore performed standard glucose tolerance tests (GTTs) and insulin tolerance tests (ITTs). Fasting baseline glucose concentrations were similar in $\Delta \mathrm{GHR}$ and control mice, but glucose excursions in $\Delta$ GHR mice were significantly higher compared with those of controls (Figure 3J). Likewise, reductions in plasma glucose levels after i.p. injection of insulin were less pronounced in $\triangle \mathrm{GHR}$ mice (Figure $3 \mathrm{~K}$ ), consistent with the development of insulin resistance. Food intake (Figure 4A) and metabolic rate, as assessed by indirect calorimetry (Figure 4, $\mathrm{B}$ and $\mathrm{C}$ ), were not significantly affected in $\triangle \mathrm{GHR}$ mice, whereas nighttime voluntary locomotor activity was lower compared with that of control animals (Figure 4D).

To examine the molecular mechanisms responsible for the development of insulin resistance in $\Delta$ GHR mice, we compared insulin-stimulated 2-deoxy- $\left[{ }^{3} \mathrm{H}\right]$ glucose (2-doG) uptake in control and $\triangle$ GHR myoblasts. Cre-mediated disruption of GHR from myoblasts resulted in a dramatic reduction ( $90 \%)$ of insulin-stimulated glucose uptake compared with that of control myoblasts (Figure 4E). Since activation of STAT5b by GH induces transcription of a number of GH target genes, including those involved in glucose and triglyceride uptake and metabolism in skeletal muscle, we assessed insulin-stimulated glucose uptake in myoblasts lacking STAT5 ( $\triangle$ STAT5ab). Uptake of 2 -doG was reduced by $30 \%$ in $\triangle$ STAT5ab myoblasts compared with controls (Figure 4F). The comparatively modest decrease (30\% vs. $90 \%$ ) suggested that GH regulates insulin responsiveness through additional mechanisms. To investigate further the mechanism for loss of insulin responsiveness in $\triangle \mathrm{GHR}$ cells, we directly examined insulin signaling components in control and $\Delta$ GHR myotubes. Immunoblotting of whole cell lysates from $\Delta$ GHR myotubes revealed a significant reduction in IR abundance compared with that of control myotubes (Figure 4G). We next examined 3 sites on IRS-1, which are known to be inhibitory to insulin signaling when phosphorylated. Basal phosphorylation of Ser 612 and Ser 636/639 was significantly decreased in $\triangle$ GHR myotubes (Figure $4 \mathrm{H}$ ), whereas phosphorylation of IRS-1 on Ser 1101 (39) was markedly increased in $\triangle$ GHR myotubes (Figure 4I). Measurement of signaling effectors downstream of IRS-1 showed that basal phosphorylation of the receptor-activated, PI-3K-mediated Akt activation loop (Thr 308) was significantly decreased in $\Delta$ GHR myotubes compared with controls (Figure 4J), consistent with the downregulation of IR. In addition, basal phosphorylation of Ser 473 on Akt, which can be activated by a number of signaling pathways, was unchanged in $\Delta$ GHR myoblasts (Figure 4J). Finally, basal Erk phosphorylation was also significantly decreased in $\Delta$ GHR myotubes compared with that of controls (Figure 4K). These results suggest that loss of GHR in skeletal muscle disrupts nutrient metabolism by desensitizing skeletal muscle to insulin action at multiple points in the IR signaling pathway.

Mice lacking IGF-1R in skeletal muscle phenocopy the skeletal muscle defects, but not the metabolic abnormalities, of $\triangle G H R$ mutants. The in vitro studies described above suggested that the effects of GH on myoblast fusion and development were largely (if not exclusively) mediated by IGF-1. To test the importance of IGF-1 signaling for skeletal muscle development in vivo, we created an additional mouse model in which the IGF-1 receptor $(\Delta \mathrm{IGF}-1 \mathrm{R}$ mice) was selectively disrupted in skeletal muscle (Supplemental Figure 2). Mice lacking the IGF-1R exhibited marked alterations in skeletal muscle development. Igf1r mRNA expression was reduced by more than $90 \%$ in the gastrocnemius muscle of $\triangle I G F-1 \mathrm{R}$ mice (Figure 5A), with no change in Ghr mRNA expression (Figure 5B). By contrast, Igf1 mRNA expression (Figure 5C) and Igf2 mRNA expression (data not shown) increased at 16 weeks. While we do not fully understand the reason for elevated Igf 1 mRNA in these mice, it is possible that compensatory pathways, such as androgens $(37,38)$, or loss of feedback inhibition of IGF-1 via autocrine effects through its receptor (40), led to increased IGF-1 production. Histomorphometric analysis of the medial gastrocnemius from 6- and 16-week-old control and $\triangle \mathrm{IGF}-1 \mathrm{R}$ mice (Figure 5, $\mathrm{D}-\mathrm{G}$ ) revealed lower myonuclei numbers (Figure 5, H and $\mathrm{K}$ ) and smaller myofibers in both type I and type II fibers in $\triangle \mathrm{IGF}-1 \mathrm{R}$ mice (Figure 5, J and M). Similar to that of $\triangle$ GHR mice, type I fibers appeared to be preferentially lost (Figure 5, I and L), although the perturbation of fiber type distribution persisted throughout all examined time points in $\triangle \mathrm{IGF}-1 \mathrm{R}$ mice.

Unlike the $\Delta$ GHR mice, which gained weight postnatally, mice lacking IGF-1R in skeletal muscle failed to attain normal body weight as they aged (Figure 5N). Analysis of body composition by $\mathrm{qMR}$ indicated $\triangle \mathrm{IGF}-1 \mathrm{R}$ mice had less fat mass at 16 weeks (Figure 5O), with no change in serum glucose (Figure 5Q), triglyceride (Figure 5R), or insulin levels (data not shown). In addition, $\triangle \mathrm{IGF}-1 \mathrm{R}$ mice failed to achieve normal lean mass by 16 weeks (Figure 5P), likely due to lesser muscle mass. In support of this notion, dual-energy X-ray absorptiometry (DXA) analysis also showed a 


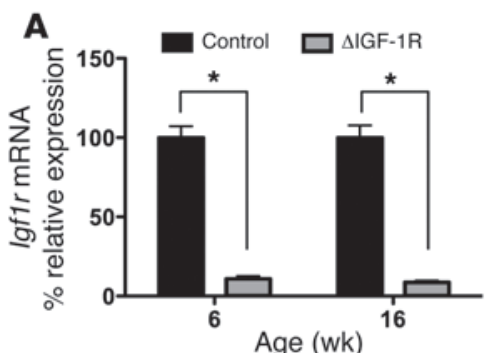

D

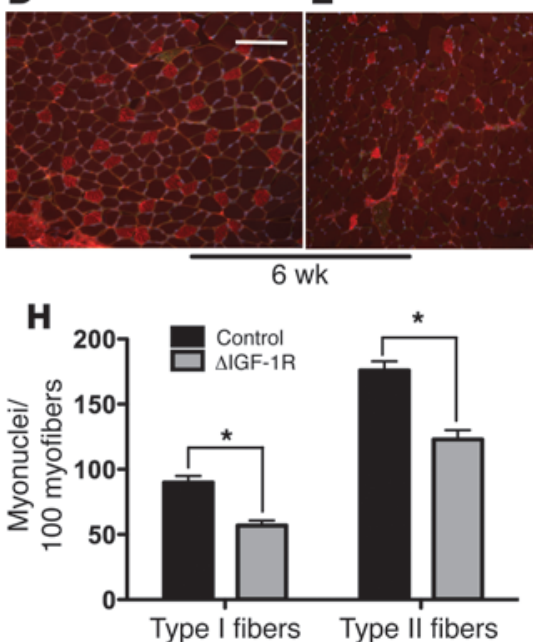

K

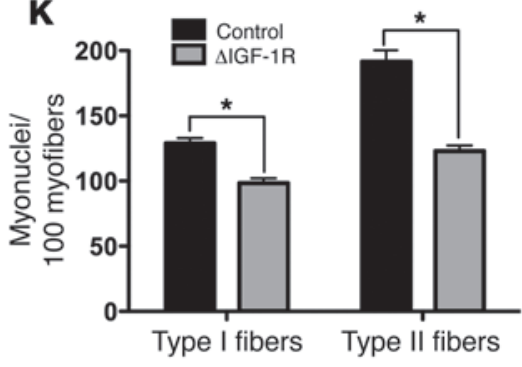

$\mathbf{N}$
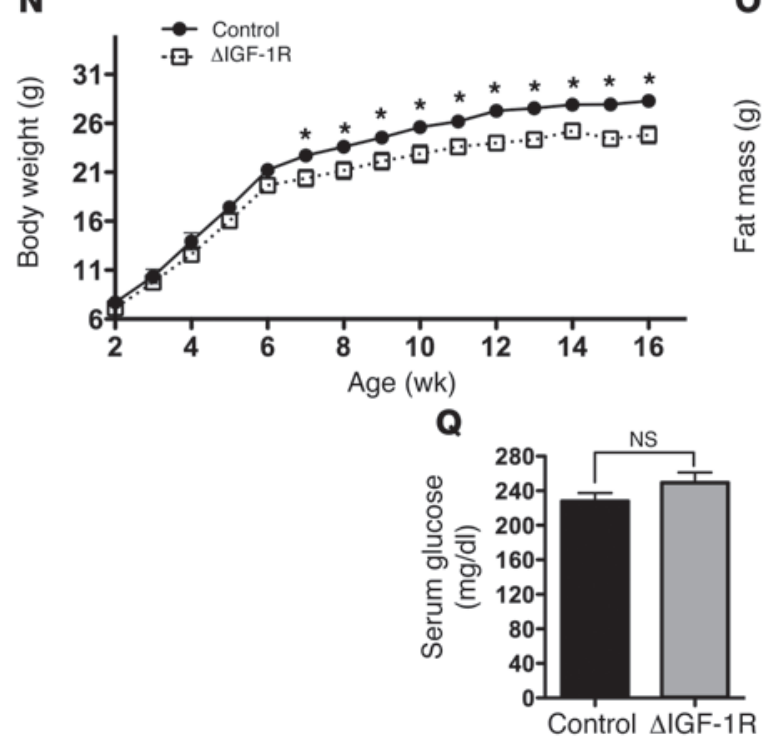

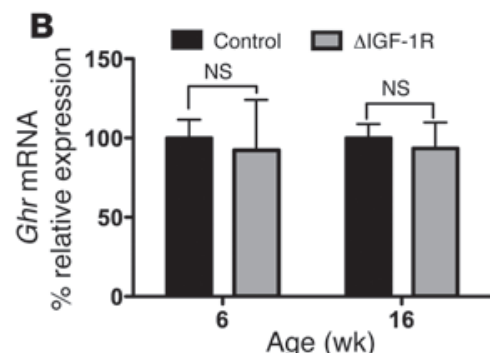

F

G
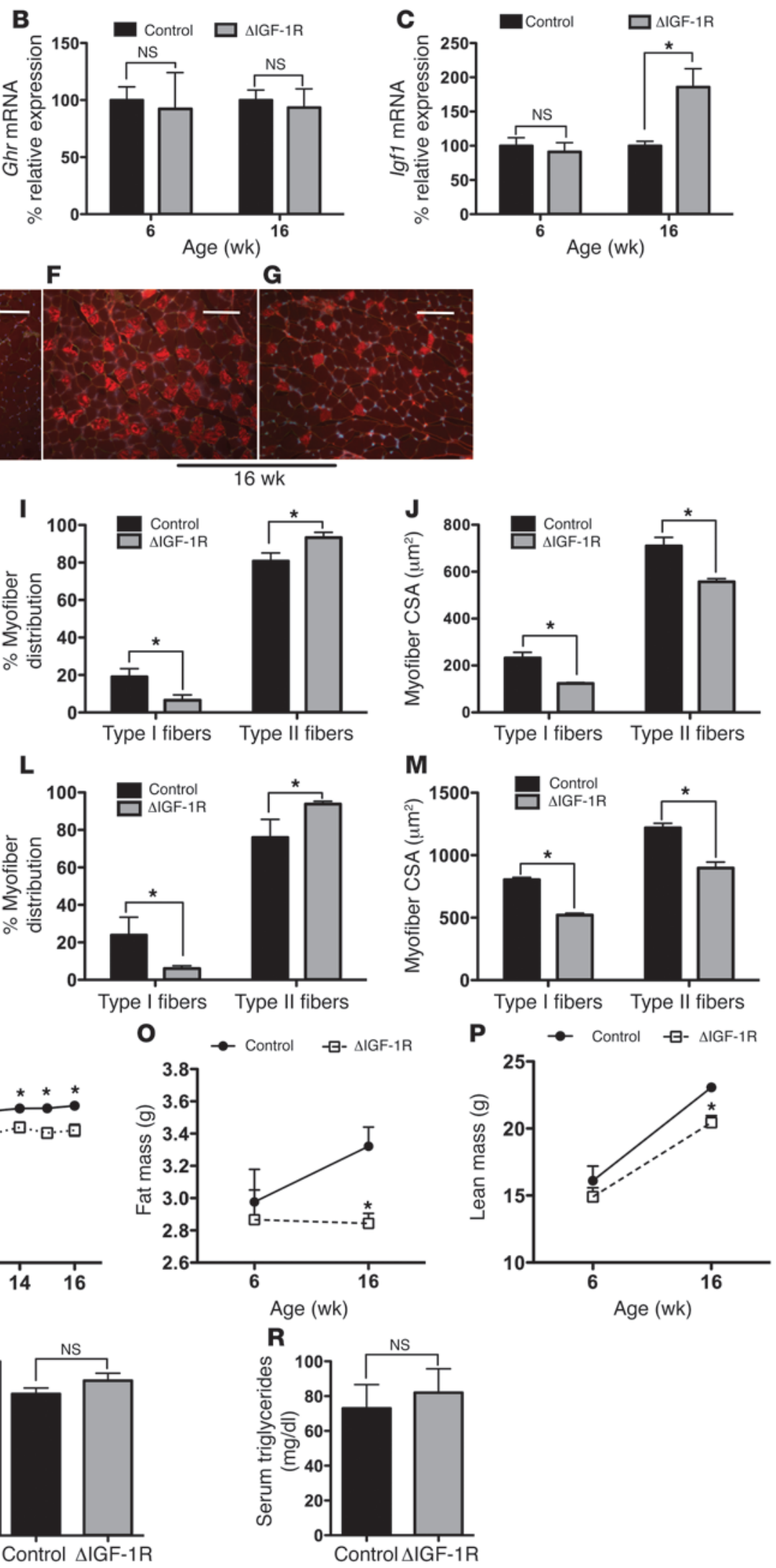


\section{Figure 5}

Mice lacking IGF-1R in skeletal muscle phenocopy the skeletal muscle defects of GHR mutants but are neither obese nor insulin resistant. mRNA was harvested from the gastrocnemius muscles of control and $\triangle \mathrm{IGF}-1 \mathrm{R}$ mice, and real-time PCR was performed using primers for (A) Igf1r, (B) Ghr, and (C) Igf1. (D-G) Sections of gastrocnemius muscle from control and $\Delta \mathrm{IGF}-1 \mathrm{R}$ mice were visualized using antibodies directed against MHC type I and laminin and counterstained with Hoechst 33258 to visualize myonuclei. Scale bars: $80 \mu \mathrm{m}$. (H-M) Histomorphometric analyses of myonuclei per 100 myofibers, percentage myofiber distribution, and myofiber diameter (CSA) were performed on sections described above for $(\mathbf{H}-\mathbf{J})$ 6-week-old and $(\mathbf{K}-\mathbf{M})$ 16-weekold control and $\Delta \mathrm{IGF}-1 \mathrm{R}$ mice. (N) Control $(n=7)$ and $\Delta \mathrm{IGF}-1 \mathrm{R}(n=10)$ mice were weighed weekly from 2 to 16 weeks of age. qMR analysis of $(\mathbf{O})$ fat and $(\mathbf{P})$ lean mass in control and $\Delta \mathrm{IGF}-1 \mathrm{R}$ mice at 6 and 16 weeks of age. Ad libitum ( $\mathbf{Q})$ serum glucose and $(\mathbf{R})$ triglyceride levels were measured in control and $\Delta \mathrm{IGF}-1 \mathrm{R}$ mice at 16 weeks of age. Unless otherwise indicated, $n=6$ for control and $\Delta \mathrm{IGF}-1 \mathrm{R}$ at all time points. Error bars indicate SEM. ${ }^{*} P<0.05$.

decrease in total body lean mass (23.073 $\mathrm{g}$ in control vs. $20.438 \mathrm{~g}$ in $\Delta$ IGF-1R mice; $n=6 ; P<0.002$ ), with no change in total body bone mineral density (BMD) in $\triangle \mathrm{IGF}-1 \mathrm{R}$ mice $\left(0.050 \mathrm{~g} / \mathrm{cm}^{2}\right.$ for control versus $0.048 \mathrm{~g} / \mathrm{cm}^{2}$ for $\Delta \mathrm{IGF}-1 \mathrm{R}$ mice; $n=6 ; P=0.131$ ). Taken together, these results indicate a disturbance of muscle development in $\Delta \mathrm{IGF}-1 \mathrm{R}$ mice, which is similar to that of $\Delta \mathrm{GHR}$ mutants, but occurs in the absence of accompanying metabolic features or insulin resistance.

\section{Discussion}

$\mathrm{GH}$ is one of the most important factors that regulates postnatal longitudinal growth, body weight, and body composition in mammals $(18,41-45)$. GH exerts anabolic actions in skeletal muscle by both promoting muscle development and facilitating nutrient uptake and utilization in the muscle, thereby coordinating global energy expenditure and body composition $(46,47)$. However, the GH and IGF-1 pathways are so intimately connected that it has been challenging to distinguish actions of GH that result secondary to GH-induced IGF-1 production, as opposed to those that might be mediated by direct GHR signaling pathways. In this study, we used a genetic approach in mice to determine the requirement of different components of the GH/IGF-1 system in skeletal muscle development and postnatal function. Understanding the precise modes and mechanisms of $\mathrm{GH}$ action in skeletal muscle is essential for developing the most effective therapies to treat sarcopenia in aging humans, cachexia associated with cancer or AIDS, or a number of other muscle-wasting conditions.

Our studies demonstrate that the GHR is required for normal skeletal muscle development and provide several lines of evidence that the effects of GH on skeletal muscle development are mediated by muscle production of IGF-1. First, acute exposure of myoblasts to GH did not affect their proliferative rate, whereas IGF-1 treatment increased both myoblast proliferation and fusion, even in cells lacking GHR. The signals generated in myoblasts, after exposure to either GH or IGF-1, appear to activate the NFATc2/ IL-4 pathway, previously shown to be critical for myoblast fusion (6). Therefore, while both GH and IGF-1 are capable of inducing NFAT translocation and activity (Figure 1, F and G), IGF-1 signaling appears to be predominate in controlling myoblast fusion and myonuclei accumulation. In accordance with this, treatment of $\triangle \mathrm{IGF}-1 \mathrm{R}$ myoblasts with $\mathrm{GH}$ failed to increase myoblast fusion, while treatment with exogenous IL-4 increased the fusion index (the percentage of fused myoblasts) but not the number of nuclei per myotube (Supplemental Figure 1, I and J). This result suggests that additional pathways, either in parallel to or downstream of NFATc 2 and IL-4 activation, might also be activated by IGF-1 to induce myoblast fusion and accumulation of additional myonuclei. For example, IGF-1 is known to activate the PI-3K/Akt pathway to inhibit GSK3 and activate mTOR/p70S6K to induce myotube hypertrophy (48). Regardless of the downstream pathways activated, IGF-1 signaling appears to be vital for GH-induced myoblast fusion. Second, elimination of the IGF-1R from myoblasts abolished GH-induced myoblast fusion. Third, the skeletal muscle phenotypes resulting from disruption of GHR and IGF-1R were strikingly similar. These findings support previous observations in mice with unrestricted disruption of IGF-1R (49) or expression of a dominant-negative IGF-1R (35), which also exhibited hypoplastic skeletal muscle. Taken together, these data provide strong evidence that $\mathrm{GH}$ actions on skeletal muscle development require local IGF-1 production. It should be noted that this conclusion is the opposite of that reached by Sotiropoulos et al. (10), who reported that GH increased skeletal muscle cell fusion but suggested that this effect was directly mediated by GH, since GH did not induce IGF-1 in their cells. The exact reasons for these different results regarding the role of IGF-1 in skeletal muscle development are unclear but may relate to the different skeletal muscle cell models and/or differences in the timing of measurements of Igf1 mRNA expression.

The histomorphometric changes observed in gastrocnemius muscles of the $\Delta$ GHR and $\Delta \mathrm{IGF}-1 \mathrm{R}$ mice are compatible with our in vitro findings and strengthen the conclusion that GH promotes skeletal muscle development through myoblast-produced IGF-1. Skeletal muscle lacking either receptor had fewer myonuclei and smaller myofiber diameters. At 6 weeks of age, type I fiber distribution was lower in both mutants, although this defect persisted only in $\triangle \mathrm{IGF}-1 \mathrm{R}$ mice at 16 weeks. These results suggest that GH normally functions to control type I fiber specification, likely through local IGF-1 production. Previous findings from mice globally deficient for the GHR exhibited significant reductions in soleus muscle type I fiber number and fiber diameter of soleus muscle (10). In addition, studies in humans have demonstrated a link between GH and fiber type specification. For example, in GH-deficient humans, the number of type I myofibers are significantly reduced in the vastus lateralis (50). These changes, together with the reduced myofiber diameters observed in the $\Delta$ GHR mice, most likely account for their reduced functional performance (grip strength and rotarod). In addition to these gross anatomical changes, it is probable that loss of GH/IGF-1 signals also compromises excitation-contraction coupling events. Consistent with this notion, preliminary assessment of showed that the force-generating capacity of single myofibers from $\Delta \mathrm{IGF}-1 \mathrm{R}$ mice was reduced compared with that of controls (data not shown). Finally, we also created a model that lacked the IGF-1 ligand, specifically in skeletal muscle, using the same mef2c-Cre mouse used to generate the $\triangle \mathrm{GHR}$ and $\triangle \mathrm{IGF}-1 \mathrm{R}$ mice. Mice lacking IGF-1 had no discernible phenotype, and their body weights were indistinguishable from control littermates at all postnatal times (data not shown). We speculate that the normal muscle development of the $\Delta \mathrm{IGF}-1$ mice might be due to compensatory activity of local anabolic factors, including IGF-2 $(51,52)$. Together, these results indicate that loss of GHR and the atten- 
dant reduction in myoblast IGF-1/IGF-1R signaling leads to deregulated fiber type specification, reduced muscle size, and subsequent compromised muscle function.

Surprisingly, loss of GHR in skeletal muscle was accompanied by a progressive increase in body weight and accumulation of peripheral fat. While nighttime voluntary locomotion was decreased in $\triangle$ GHR mice, food intake decreased and metabolic rate tended to increase in the mutants. We therefore assumed that the decreased nocturnal activity was likely not the causative factor for the observed increase in fat mass. Rather, we suspected that loss of GHR from skeletal muscle caused alterations of global nutrient metabolism. Indeed, serum glucose in the fed state was elevated in $\triangle$ GHR mutants, and the development of insulin resistance in these mice was confirmed by GTTs and ITTs. This finding might seem paradoxical, given that global GH resistance in mice that lack GHR/GH binding protein results in increased insulin sensitivity (53-59). However, GHR/GH binding protein mutant mice exhibit increased liver IR abundance and enhanced hepatic insulin-stimulated IR tyrosine phosphorylation (59-61), which likely accounts for their increased insulin sensitivity. Therefore, it would appear that the different metabolic profile exhibited by our $\triangle$ GHR mice, which lack GHR exclusively in skeletal muscle, is explained partially by maintenance of insulin action in nonskeletal muscle tissues in which GHR remains intact. It should also be noted that serum insulin remained unchanged in $\triangle$ GHR mice at all time points examined, further supporting normal insulin action and regulation in other insulin-responsive tissues. Taken together, the increased serum glucose and triglyceride levels observed in $\triangle$ GHR mice, in the face of normal insulin levels, suggests a failure of nutrient uptake by skeletal muscle. We predict that the increased fat mass observed in $\triangle$ GHR mutants results from redistribution of this nutrient supply from skeletal muscle to GHR-intact adipose tissue.

Importantly, the body composition and metabolic alterations seen in $\triangle$ GHR mice were not observed in $\triangle \mathrm{IGF}-1 \mathrm{R}$ mice, which had reduced body weights, decreased peripheral fat, and normal, nonfasting serum glucose levels. These findings clearly suggest that the changes in body composition and insulin resistance seen in the $\Delta$ GHR mice are not due to loss of IGF-1 signaling in muscle. Rather, the actions of IGF-1 seem to be restricted primarily to anatomical development of the skeletal muscle. Interestingly, mice rendered IGF-1 resistant in skeletal muscle by targeting overexpression of a dominant-negative IGF-1R in skeletal muscle (MKR mice), exhibited metabolic disturbances similar to those observed in our $\Delta$ GHR mice, including increased peripheral fat and insulin resistance (62). In the MKR mice, expression of the dominant-negative IGF-1R effectively disrupts both IGF-1R and IR signaling by inducing formation of nonfunctioning hybrids between the mutant and the endogenous IGF-1 and IRs. By contrast, we propose that insulin signaling is attenuated in the $\triangle$ GHR mice due to decreased IR abundance and increased phosphorylation of IRS-1 on Ser 1101 (39), which together inhibit insulin signaling. Thus, the MKR mice and $\triangle$ GHR mice share a similar metabolic phenotype as a result of compromised IR signaling in skeletal muscle, which is manifested through distinct mechanisms. These defects in insulin signaling most likely represent the primary reason for the development of insulin resistance and increased peripheral adiposity in both models.

Our studies also shed light on the molecular mechanisms responsible for the development of insulin resistance in skeletal muscle from the $\triangle$ GHR mice. Since myoblasts lacking STAT5ab were also less sensitive to insulin-induced glucose uptake, it would appear that GH modulates insulin sensitivity, at least in part, through STAT5. Indeed, a number of genes modulating glucose and triglyceride uptake and metabolism are known to be regulated through GH-induced STAT5 activation (63). Accordingly, mice lacking STAT5ab in skeletal muscle also had increased fat mass, further suggesting the involvement of GH-induced STAT5mediated genes in the metabolic effects seen in our $\triangle$ GHR mice $(64,65)$. However, the reduction of insulin-induced glucose uptake seen in $\triangle$ STAT5ab myoblasts was not as pronounced as that of $\triangle$ GHR myoblasts, indicating the contribution additional pathways in the development of insulin resistance. As mentioned above, insulin resistance in myotubes lacking GHR appears to derive from changes at a number of proximal and distal points along the insulin signaling pathway, including decreased IR abundance, reduced basal Akt (Thr 308) and Erk phosphorylation, and the increased Ser 1101 phosphorylation of IRS-1 already discussed. Basal phosphorylation of other known inhibitory IRS-1 residues (Ser 612 and Ser 636/639) was reduced, at least in the context of our in vitro model system. However, it remains possible that the hyperglycemic, hypertriglyceridemic, and likely inflammatory conditions experienced in vivo may further exacerbate insulin resistance in skeletal muscle through pathways known to act on these inhibitory IRS-1 residues $(66,67)$. Since IR signals primarily through the PI-3K pathway, it is more likely that the reduction in basal Erk activation is a result of GHR disruption. However, loss of GH-mediated Erk activity could have indirect effects to further suppress insulin-induced glucose uptake in $\triangle \mathrm{GHR}$ myoblasts. Erk interacts with TSC2 (68) to relieve its suppression of mTOR, allowing GLUT expression and glucose uptake (69), and the ability of $\mathrm{GH}$ to alter insulin sensitivity via nutrient-sensing pathways, like $\mathrm{PI} 3 \mathrm{~K} / \mathrm{mTOR} / \mathrm{S} 6 \mathrm{~K}(70)$, is currently being investigated.

In conclusion, the results presented in this paper indicate 2 circumscribed roles for GHR signaling is skeletal muscle. First, by regulating myoblast production of IGF-1, GH promotes normal myofiber type specification, myonuclei accumulation, and expansion of myofiber diameter, processes which are required for development of fully functional skeletal muscle. Second, GH functions independent of IGF-1R signaling to facilitate normal insulin action in skeletal muscle, which ultimately impacts global nutrient metabolism. Precisely how these distinct GH-generated signals are compartmentalized within skeletal muscle is a question currently under investigation in our laboratory. Further elucidation of these mechanisms should guide more informed use of GH or GH analogs for promoting muscle development and attenuating muscle loss as well as provide alternative means to affect insulin sensitivity and global nutrient metabolism across a range of metabolic syndromes.

\section{Methods}

Primary myoblast isolation and culture. Primary myoblast cultures were prepared from tibialis anterior and gastrocnemius muscles of 4-week-old wildtype, floxed GHR, and IGF-1R mice and purified to more than $99 \%$ as previously described (36). Growth media (GM) consisted of Ham's F-10, 20\% FBS, $5 \mathrm{ng} / \mathrm{ml}$ basic recombinant human FGF (bFGF) (Promega), $100 \mathrm{U} / \mathrm{ml}$ penicillin G (GIBCO BRL), and $100 \mu \mathrm{g} / \mathrm{ml}$ streptomycin (GIBCO BRL). Myoblasts were grown in type I collagen-coated (BD Biosciences) plates. Fusion was induced by growing myoblasts in Entactin-Collagen-Laminincoated (ECL-coated) (Upstate Biotechnology) dishes and low-serum differentiation media (DM) (DMEM with 1\% insulin-transferrin-selenium-A 
supplement [GIBCO BRL]). For in vitro deletion of the GHR, myoblasts with the floxed GHR alleles were cultured to be $80 \%$ confluent and then infected with an adenovirus encoding Cre recombinase (adenoCre) (Vector Biolabs) at a titer of $100 \mathrm{MOI}$ in a small volume of sterile PBS. Myoblasts infected with 100 MOI of adenovirus encoding GFP (adenoGFP) (Vector Biolabs) were used as control. One hour after infection with adenovirus, GM were added to myoblasts and cultured for the next 48 hours. GHR deletion was confirmed for every infection by real-time PCR and immunoblotting with anti-GHR antibody (71). AdenoGFP-infected (referred to as control) or adenoCre-infected (referred to as $\Delta \mathrm{GHR}$ ) primary myoblasts were replated in 6-well plates for proliferation and fusion assays. $\triangle \mathrm{IGF}-1 \mathrm{R}$ and $\triangle$ STAT5 myoblasts were generated in an analogous manner.

Proliferation and fusion assays. Myoblasts were plated in 6-well type I collagen-coated plates at low cell density $\left(1 \times 10^{5}\right.$ cells/well of a 6 -well plate $)$ and cultured in Ham's F-10 media with 1\% FBS for 10 hours to arrest the cells in $\mathrm{G}_{0}$ phase. Cells were treated with $500 \mathrm{ng} / \mathrm{ml} \mathrm{GH}, 100 \mathrm{ng} / \mathrm{ml} \mathrm{IGF-1,} \mathrm{or} 20 \%$ serum and $5 \mathrm{ng} / \mathrm{ml} \mathrm{bFGF}$ and pulsed with $10 \mu \mathrm{M} \mathrm{BrdU}$ for the next 8 hours. Myoblasts were stained for anti-BrdU-APC and 7-amino-actinomycin D (APC BrdU Flow Kit, BD Biosciences) for proliferation and analyzed by FACSCalibur (Becton Dickinson). A total of 10,000 events were collected for each sample, and results were analyzed with WinMDI version 2.8. For fusion assays, after 24 or 48 hours in DM, myoblasts were fixed in 3.7\% formaldehyde for 10 minutes. Cell membranes were identified by staining with $10 \mu \mathrm{M}$ 1,1-dioctadecyl-3,3,3', $3^{\prime}$-tetramethylcarbocyanine perchlorate (DiI), while nuclei were identified by staining with $300 \mathrm{nM}$ DAPI. The number of nuclei were counted and expressed as the number per DiI-labeled myotube.

Real-time PCR analysis. Total RNA was extracted from cells using the TRIzoL method, as recommended by the manufacturer (catalog 15596-026, Invitrogen). RNA concentration was estimated spectrophotometrically, and only pure RNA ( $A_{260} / A_{280}$ ratio of 1.8$)$ was used for further analysis. First-strand cDNA was synthesized using the iScript cDNA Synthesis Kit (Bio-Rad Laboratories). The cDNA was amplified in the Opticon Continuous Fluorescent Detector (MJ Research) using iQ SYBR Green Supermix (Bio-Rad) and sequence specific primers according to the manufacturer's instructions. A total of $1 \mu \mathrm{g}$ RNA was used in each reaction and combined with specific primers. Primer sequences used were as follows: $G h r$, forward 5'-AGCCTCGATTCACAAGTGTCG-3', reverse 5'-GATGACCAATTCTTGCAGCTTG-3'; Igf1r, forward 5'-TTGTGTTGTTCGTCCGGTGTG-3', reverse 5'-ATGTGCCCAAGTGTGTGCG-3'; Igf1, forward 5'-GCTCTTCAGTTCGTGTGTGGAC-3', reverse 5'-TTGGGCTGTCAGTGTGGCGC-3'; Actb, forward $5^{\prime}$-ACCTCCTACAATGAGCTGC-3', reverse 5'-TGCCAATAGTGATGACCT-3'; Pax7, forward 5'-CACCCCGGGGACAGAGGAAGAT-3', reverse 5'-GAGAGGGCGGGGTACAAGGAAGAC-3'; Myf5, forward 5'-AGCCCACCAGCCCCACCTC-3', reverse 5'-TTCTGCCCAGCTTGTCTTTCCTTC3'; Cdh15, forward 5'-CATCCCACCCATTAGTGTGTC-3', reverse 5'-TCCCAGTGAACTTGTCGATAGA-3'; Des, forward 5'-GTGGATGCAGCCACTCTAGC-3', reverse 5'-TTAGCCGCGATGGTCTCATAC-3'; Myod1, forward 5'-ACACTTCTGGCCCGAGGTAT-3', reverse 5'-GACTTCCAATGTTTCATCAGTGC-3'; Myog, forward 5'-GAGACATCCCCCTATTTCTACCA-3', reverse 5'-GCTCAGTCCGCTCATAGCC-3'; Myb3, forward 5'-CCTTGGCACCAATGTCCCGGCTC-3', reverse 5'-GAAGCGCAATGCAGAGTCGGTG-3'; Il4, forward 5'-AACCCCCAGCTAGTTGTCATCCTG3', reverse 5'-CATCGAAAAGCCCGAAAGAGTCTC-3'. PCR was performed in triplicate for each CDNA, and results were averaged and normalized to endogenous $\beta$-actin reference transcripts.

Cell lysis and immunoblot analysis. To collect cell lysates for immunoblot analysis, myoblasts were washed twice with ice-cold PBS and resuspended in cell lysis buffer (50 mM Tris, pH 7.4, $150 \mathrm{mM} \mathrm{NaCl}, 1 \mathrm{mM} \mathrm{MgCl}$, 1 mM EDTA, $1 \%$ Triton $\mathrm{X}-100$, and $10 \%$ glycerol) containing protease and phosphatase inhibitors (Sigma-Aldrich). Cell lysates were homogenized by rotating at $4^{\circ} \mathrm{C}$ for 30 minutes and then centrifuged at $8,700 \mathrm{~g}$ for 20 minutes at $4^{\circ} \mathrm{C}$. Supernatant was transferred to microcentrifuge tubes, and protein concentration was measured using Bradford protein assay (Bio-Rad). For immunoblotting of whole cell lysates, equal amounts of protein $(10 \mu \mathrm{g} / \mathrm{lane})$ were solubilized in Laemmli sample buffer and loaded onto a mini-SDS-PAGE system. Following electrophoresis, proteins were transferred to a polyvinylidene difluoride membrane using a Bio-Rad wet transfer system. Protein transfer efficiency was verified using prestained protein markers. Membranes were blocked with $5 \%$ nonfat dry milk for 1 hour at room temperature and subsequently incubated overnight at $4^{\circ} \mathrm{C}$ with antibodies directed against the protein of interest. Signals were detected using a horseradish peroxidase-conjugated secondary antibody, and bound antibodies were visualized using the Supersignal West Dura Substrate (Pierce). Western blot photographic results were scanned with a Canon flatbed scanner. Proteins in the cytoplasm and nuclear fractions were separated using NE-PER nuclear and cytoplasmic extraction reagents according to the manufacturer's instructions (Pierce Biotechnology Inc.). Antibodies used were as follows: anti-IGF-1R $\beta$-subunit (C-20), anti$\beta$-actin (C4), and anti- $\alpha$-tubulin all from Santa Cruz Biotechnology Inc.; anti-GHR (AL47) antibody (71); and anti-phospho-Erk1/2, anti-Erk1/2, antiIR, anti-phospho-IRS-1 (Ser 1101, Ser 612, and Ser 636/639), anti-IRS-1, anti-phospho-Akt (Thr 308 and Ser 473), and anti-Akt all from Cell Signaling Technology. Horseradish peroxidase-conjugated rabbit and mouse secondary antibodies were obtained from Pierce Biotechnology Inc. Polyvinylidene difluoride membrane, Laemmli sample buffer, and other electrophoresis supplies were obtained from Bio-Rad. Densitometry was performed using the analysis feature of Adobe Photoshop CS4. Briefly, images were converted from 8-bit RGB to grayscale, and a single, uniform selection box was used to collect mean gray density values for each band of interest. These values were normalized to similar measurements performed for relevant loading control proteins. Quantitation from multiple experiments $(n \geq 3)$ was graphed, and the results are presented with representative blots.

NFAT luciferase reporter assay. For transient transfections, primary myoblasts were grown in 6-well plates at $2.5 \times 10^{5}$ cells per well. For each well, $2 \mu \mathrm{g}$ NFAT reporter construct DNA (a gift from Majd Zayzafoon, University of Alabama at Birmingham) was complexed with $2 \mu \mathrm{l}$ FuGENE HD Transfection Reagent (Roche) for 10 minutes at room temperature in a total volume of $500 \mu \mathrm{l}$ of Ham's F10 media. The DNA-FuGENE mixture was added to the wells containing $2 \mathrm{ml}$ of Ham's F10 media and incubated overnight at $37^{\circ} \mathrm{C}$. Twenty-four hours after transient transfection with the NFAT reporter construct, myoblasts were plated at $7.5 \times 10^{5}$ cells per well of the ECL-coated 24-well plate. Myoblasts were allowed to settle, and the medium was replaced with DM to induce fusion for a duration of 24 hours. Following this, cells were treated with $500 \mathrm{ng} / \mathrm{ml}$ bovine GH or $100 \mathrm{ng} / \mathrm{ml} \mathrm{IGF-1} \mathrm{or}$ $1 \mu \mathrm{M}$ ionomycin for 5 hours and assayed for luciferase expression.

Measurement of 2-doG uptake. Myoblasts were plated in 12 -well plates $\left(1 \times 10^{5}\right.$ cells/well) and cultured in Ham's F-10 with 20\% FBS for 5 hours. Myoblasts were serum starved in $0.1 \%$ Ham's F-10 media for 12 hours and washed with Krebs-Ringer phosphate HEPES buffer (KRPH buffer, $136 \mathrm{mM} \mathrm{NaCl}, 4.7 \mathrm{mM}$ $\mathrm{KCl}, 1.25 \mathrm{mM} \mathrm{MgSO}_{4}, 1.25 \mathrm{mM} \mathrm{CaCl}_{2}$, and $20 \mathrm{mM}$ HEPES, pH 7.4) in $0.2 \%$ BSA. Cells were then incubated with or without $10 \mathrm{nM}$ insulin in $900 \mu \mathrm{l}$ of $\mathrm{KRPH}$ buffer in $0.2 \% \mathrm{BSA}$ for 1 hour at $37^{\circ} \mathrm{C}$. For $\left[{ }^{3} \mathrm{H}\right] 2$-doG uptake, $50 \mu \mathrm{l}$ reaction mixture, containing $5 \mu \mathrm{Ci} 2-\left[1,2-{ }^{3} \mathrm{H}\right]$-deoxy-D-glucose (PerkinElmer) and $1 \mathrm{mM} 2$-doG, was added to each well for 3 minutes at room temperature. The reaction was stopped by washing cells 3 times in ice-cold KRPH buffer containing $0.3 \mathrm{mM}$ phloretin. Next, cells were lysed using $500 \mu \mathrm{l} 1 \mathrm{~N} \mathrm{NaOH}$ at $50^{\circ} \mathrm{C}$ for 30 minutes, followed by addition of $500 \mu \mathrm{l} 1 \mathrm{~N} \mathrm{HCl}$ to neutralize $\mathrm{NaOH}$. The incorporated radioactivity was determined by liquid scintillation counting, and specific uptake (i.e., the background subtracted from the total uptake) was obtained. Assays were performed in triplicate, and data are presented as picomoles of 2 -doG per milligram protein per minute. 
Generation of skeletal muscle-specific GHR and IGF-1R ligand knockout mice. To generate the knockout mice, GHR $(72)$ or $\operatorname{IGF}-1 \mathrm{R}(31,73)$ homozygous floxed mice ( $G h r^{f / / f l}$ or $I g f 1 r^{f / / f l}$ mice) were crossed with mice that express the Cre recombinase specifically in skeletal muscle, under the control of the mef-2c-73k promoter (heterozygous mef- $2 \mathrm{c}-73 \mathrm{kCre}{ }^{+/-}$mice) (a gift from Brian Black, University of California San Francisco, San Francisco, California, USA).The F1 transheterozygote progeny from this mating (i.e., Ghrfl/ /mef-2c-73kCre $\mathrm{k}^{+/-}$mice) were then crossed with the homozygous floxed animals (i.e., Ghrl/fl mice) to produce the Cre-positive knockout animals ( $\triangle$ GHR or $\triangle \mathrm{IGF}-1 \mathrm{R}$ mice) and Cre-negative littermate controls (i.e., $\mathrm{Ghr}^{l / f l} / \mathrm{mef}-2 \mathrm{c}-73 \mathrm{kCre}^{+/-}$and $\mathrm{G} h \mathrm{r}^{l / f l} / \mathrm{mef}-2 \mathrm{c}-73 \mathrm{kCre}^{-/-}$mice, respectively). All animals were on a mixed genetic background of $129 \mathrm{SVJ}$ and C57BL/6. Mice were fed ad libitum and weighed weekly. All studies were performed in male mice. The Institutional Animal Care and Use Committee of the University of Alabama at Birmingham approved all animal work.

Histomorphometric analysis. Gastrocnemius muscles were embedded in OCT-embedding media, and mounts were cut in $5-\mu \mathrm{m}$ serial sections at $-22^{\circ} \mathrm{C}$ using a Leica CM1900 cryostat microtome. Slides were kept in a humidified chamber throughout the staining protocol. Sections were fixed for 45 minutes at room temperature in 3\% neutral-buffered formalin and washed twice for 5 minutes with $1 \mathrm{x}$ PBS (all subsequent PBS wash steps were $3 \times 5$ minutes). Sections were blocked with $5 \%$ goat serum in PBS for 20 minutes at room temperature, followed by a wash step. Primary and secondary antibodies were diluted in $1 \%$ goat serum in PBS. Anti-MHC type I (anti-MHC I) primary antibody (mouse mAb NCL-MHCs, NovoCastra Laboratories; $1: 100$ ) was applied for 30 minutes at $37^{\circ} \mathrm{C}$. After a wash step, sections were incubated with Alexa Fluor 594-conjugated goat anti-mouse secondary antibody (Pierce Biotechnology Inc.; 1:200) for 30 minutes at $37^{\circ} \mathrm{C}$. Sections were washed and again blocked ( $5 \%$ goat serum in PBS) for 20 minutes at room temperature. To locate sarcolemma for myofiber sizing, a wash step was followed by incubation with anti-laminin mouse $\mathrm{mAb}$ (VP-L551, NovoCastra Laboratories; $1: 80$ ) for 30 minutes at $37^{\circ} \mathrm{C}$, a wash step, and incubation with Alexa Fluor 488-conjugated goat anti-mouse secondary antibody (Pierce Biotechnology Inc.; 1:200) for 30 minutes at $37^{\circ} \mathrm{C}$. Nuclei were revealed using a Hoecsht 33258 DNA counterstain (Molecular Probes; 1:10,000 in PBS) for 2 minutes at room temperature. Slides underwent a final aspiration and were mounted with $1 \%$ paraphenylene diamine and $90 \%$ glycerol in PBS. Slides and coverslips were bound together using nail polish and stored, protected from light, at $-20^{\circ} \mathrm{C}$.

Immunofluorescence microscopy. High-resolution (48-bit TIFF) images were captured at magnifications of $\times 10$ and $\times 20$, using an Olympus MagnaFire SP camera (S99810) and MagnaFire SP (http://download.optronics.com/ 02-camera-download-details.asp?ProductTable_ID=23) with an Olympus BX51 fluorescent microscope. Image analysis was performed using ImagePro Plus 5.0 software. All analyses were conducted by a single analyst, who was blinded to the age and the genotype of each sample. Myofiber-type distribution was determined from $935 \pm 47$ myofibers per sample. Myofibers negative for MHC I were classified as type II fibers. For cross-sectional area (CSA) measurements, each myofiber was manually traced along its laminin-stained border. CSA (in $\mu \mathrm{m}^{2}$ ) was calibrated using a stage micrometer, and only those fibers determined to be cross-sectional based on a roundness factor of less than 1.639 were included in the analysis (roundness = perimeter ${ }^{2} / 4_{\pi}$ area; perfect circle $=1.0$, pentagon $=1.163$, square $=1.266$, equilateral triangle $=1.639$ ).

Assessment of body composition. All body composition scans were performed in the morning. Mice were anesthetized with isoflurane (2\%), and body composition was assessed in vivo using DXA (GE Lunar PIXImus software version 1.45 , Lunar) at 6,16 , and 26 weeks. Fat mass and soft lean tissue mass were determined as described previously (74). Briefly, mice were anesthetized and placed in a prostrate position on the imaging plate, and a total body scan was done in approximately 5 minutes. All analyses were done excluding the head. qMR was performed without sedation in less than 90 seconds for the noninvasive determination of fat and lean mass (3-in-1 Composition Analyzer, Echo Medical Systems).

Analysis of skeletal muscle function. For the grip strength test, mice were held by the base of the tail above a wire grid and gently lowered down until all 4 paws grasped the grid. Mice were then maneuvered to a horizontal position and gently pulled by the tail until they released the grip. Maximal force achieved by the animal was recorded for 3 successive trials and averaged. For the rotarod test, the rotating drum was set to accelerate from 4 to $40 \mathrm{rpm}$ over 300 seconds; 30 minutes were left between training and test sessions and 15 minutes were left between each test session. Speed of rotation was constant upon reaching $40 \mathrm{rpm}$. Three trials were performed, and duration on the rotating rod and rotating distance measured were averaged.

Necropsy and tissue processing. Mice were sacrificed at 6, 16, or 26 weeks and whole body necropsy was performed. Fat pads (inguinal, gonadal, retroperitoneal, subcutaneous, mesenteric, and brown adipose tissue), livers, and hearts were removed and weighed. Gastrocnemius, soleus, and tibialis anterior muscles were dissected, weighed and snap frozen in liquid nitrogen, and stored at $-80^{\circ} \mathrm{C}$. Blood was allowed to clot at room temperature, and serum was separated by centrifugation and stored at $-80^{\circ} \mathrm{C}$ until further analysis.

Serum analysis. Triglyceride, insulin, and glucose levels were measured in $10 \mu \mathrm{l}$ samples of serum with the Ektachem DT II System (Johnson \& Johnson Clinical Diagnostics). Total nonesterified FFAs were measured using an enzymatic, colorimetric method ("NEFA-C" reagents, Wako Diagnostics) modified to a microplate format.

Energy expenditure, locomotor activity tests, and food intake. Total energy expenditure (TEE) and locomotor activity for individually housed mice were analyzed using an indirect-calorimetric system (Labmaster, TSE Systems GmbH). Mice were placed in air-tight respiratory chambers with a flow rate of $0.45 \mathrm{l} / \mathrm{h}$ and placed at a constant temperature $\left(22.0^{\circ} \mathrm{C} \pm 1.0^{\circ} \mathrm{C}\right)$. Energy expenditure was calculated using 2-minute sample $\mathrm{O}_{2}$ and $\mathrm{CO}_{2}$ concentrations for quantification of $\mathrm{O}_{2}$ consumption and $\mathrm{CO}_{2}$ production. TEE was determined by calculating the average hourly energy expenditure over 22 hours and then multiplied by 24 . Resting energy expenditure (REE) was calculated by averaging the 3 lowest 10-consecutive-minute periods of energy expenditure, with at least 1 hour between each period. This was then multiplied to generate a 24-hour REE. Detection of locomotor activity within the chambers was performed with an infrared sensor arranged in a grid pattern for horizontal ( $x$, y level) activity. Movement was monitored continuously and recorded by the computer every 9 minutes. Locomotor activity was calculated by total counts every 9 minutes and expressed as counts per 24 hours. All above experiments were performed under red light to avoid changes in circadian rhythmic activity. Food consumption was determined by measuring the loss of food from the hopper of each animal over a 3-day period.

Assessment of glucose disposal and insulin sensitivity. For ITTs, mice were fasted for 4 hours and injected with insulin ( $0.2 \mathrm{U} / \mathrm{kg}$ body weight) i.p. Blood was collected from the tail vein and monitored for glucose, using blood glucose strips and a glucometer every 15 minutes for 2 hours. ITT data were calculated as percentage of initial blood glucose concentration. For GTTs, mice were injected with glucose $(1.5 \mathrm{~g} / \mathrm{kg}$ body weight $)$ i.p. after an overnight fast ( 16 hours), and blood glucose levels were measured every 15 minutes for 2 hours.

Statistics. All statistical analysis was performed using the Student's $t$ test (2-tailed). Error bars represent SEM. $P$ values of less than 0.05 are considered to be statistically significant.

\section{Acknowledgments}

We are grateful for the assistance of Grace Pavlath in establishing primary muscle cultures and providing helpful suggestions during 
the completion of this work. This work was supported by grants from the NIH (R01DK46395 to S.J. Frank and P30NS47466 to T. van Groen) and a Veterans Administration Research Career Scientist Award (to T.L. Clemens).

Received for publication January 26, 2010, and accepted in revised form August 18, 2010.
Address correspondence to: Thomas L. Clemens, 601 N. Caroline St., JHOC 5242, Baltimore, Maryland 21287-0882, USA. Phone: 410.955.3245; Fax: 410.614.1451; E-mail: tclemen5@jhmi.edu.

Mahendra D. Mavalli's present address is: Department of Pathology, University of Alabama at Birmingham, Birmingham, Alabama, USA.
1. Kablar B, et al. MyoD and Myf-5 define the specification of musculature of distinct embryonic origin. Biochem Cell Biol. 1998;76(6):1079-1091.

2. Hasty $P$, et al. Muscle deficiency and neonatal death in mice with a targeted mutation in the myogenin gene. Nature. 1993;364(6437):501-506.

3. Nabeshima Y, Hanaoka K, Hayasaka M, Esumi E, Li $\mathrm{S}$, Nonaka I. Myogenin gene disruption results in perinatal lethality because of severe muscle defect. Nature. 1993;364(6437):532-535.

4. Horsley V, Pavlath GK. Forming a multinucleated cell: molecules that regulate myoblast fusion. Cells Tissues Organs. 2004;176(1-3):67-78.

5. Horsley V, Friday BB, Matteson S, Kegley KM, Gephart J, Pavlath GK. Regulation of the growth of multinucleated muscle cells by an NFATC2-dependent pathway. J Cell Biol. 2001;153(2):329-338.

6. Horsley V, Jansen KM, Mills ST, Pavlath GK. IL-4 acts as a myoblast recruitment factor during mammalian muscle growth. Cell. 2003;113(4):483-494.

7. Baum HB, et al. Effects of physiologic growth hormone therapy on bone density and body composition in patients with adult-onset growth hormone deficiency. A randomized, placebo-controlled trial. Ann Intern Med. 1996;125(11):883-890.

8. Ullman M, Oldfors A. Effects of growth hormone on skeletal muscle. I. Studies on normal adult rats. Acta Physiol Scand. 1989;135(4):531-536.

9. Weber MM. Effects of growth hormone on skeletal muscle. Horm Res. 2002;58 suppl 3:43-48.

10. Sotiropoulos A, et al. Growth hormone promotes skeletal muscle cell fusion independent of insulinlike growth factor 1 up-regulation. Proc Natl Acad SciUS A. 2006;103(19):7315-7320.

11. Kaplan S, Kostyo JL. Hormonal regulation of growth and metabolic effects of growth hormone. In: Kostyo JL, ed. Handbook of Physiology. Vol. 5. New York, New York, USA: Oxford University Press; 1999:129-143.

12. Zhang Y, Jiang J, Kopchick JJ, Frank SJ. Disulfide linkage of growth hormone $(\mathrm{GH})$ receptors (GHR) reflects $\mathrm{GH}$-induced $\mathrm{GHR}$ dimerization. Association of JAK2 with the GHR is enhanced by receptor dimerization.J Biol Chem. 1999;274(46):33072-33084.

13. Argetsinger LS, et al. Identification of JAK2 as a growth hormone receptor-associated tyrosine kinase. Cell. 1993;74(2):237-244.

14. Miller WL, Eberhardt NL. Structure and evolution of the growth hormone gene family. Endocr Rev. 1983;4(2):97-130.

15. Carter-Su C, Schwartz J, Smit LS. Molecular mechanism of growth hormone action. Annu Rev Physiol. 1996;58:187-207

16. Davey HW, Xie T, McLachlan MJ, Wilkins RJ, Waxman DJ, Grattan DR. STAT5b is required for GHinduced liver IGF-I gene expression. Endocrinology. 2001;142(9):3836-3841.

17. Woelfle J, Billiard J, Rotwein P. Acute control of insulin-like growth factor-I gene transcription by growth hormone through Stat5b.J Biol Chem. 2003;278(25):22696-22702.

18. Lupu F, Terwilliger JD, Lee K, Segre GV, Efstratiadis A. Roles of growth hormone and insulin-like growth factor 1 in mouse postnatal growth. Dev Biol. 2001;229(1):141-162.

19. Liu JL, Yakar S, LeRoith D. Conditional knockout of mouse insulin-like growth factor-1 gene using the Cre/loxP system. Proc Soc Exp Biol Med. 2000;223(4):344-351.
20. Yakar S, Liu JL, Le Roith D. The growth hormone/ insulin-like growth factor-I system: implications for organ growth and development. Pediatr Nephrol. 2000;14(7):544-549.

21. Le RD, Bondy C, Yakar S, Liu JL, Butler A. The somatomedin hypothesis: 2001. Endocr Rev. 2001;22(1):53-74.

22. Yakar S, Wu Y, Setser J, Rosen CJ. The role of circulating IGF-I: lessons from human and animal models. Endocrine. 2002;19(3):239-248.

23. DiGirolamo DJ, et al. Mode of growth hormone action in osteoblasts. J Biol Chem. 2007; 282(43):31666-31674.

24. Adams GR, Haddad F. The relationships among IGF-1, DNA content, and protein accumulation during skeletal muscle hypertrophy. J Appl Physiol. 1996;81(6):2509-2516.

25. Goldspink G. Changes in muscle mass and phenotype and the expression of autocrine and systemic growth factors by muscle in response to stretch and overload. J Anat. 2010;194(pt 3):323-334.

26. Musaro A, et al. Localized Igf- 1 transgene expression sustains hypertrophy and regeneration in senescent skeletal muscle. Nat Genet. 2001;27(2):195-200.

27. Owino V, Yang SY, Goldspink G. Age-related loss of skeletal muscle function and the inability to express the autocrine form of insulin-like growth factor-1 (MGF) in response to mechanical overload. FEBS Lett. 2001;505(2):259-263.

28. Favelyukis S, Till JH, Hubbard SR, Miller WT. Structure and autoregulation of the insulin-like growth factor 1 receptor kinase. Nat Struct Biol. 2001;8(12):1058-1063.

29. Ullrich A, et al. Insulin-like growth factor I receptor primary structure: comparison with insulin receptor suggests structural determinants that define functional specificity. EMBO J. 1986;5(10):2503-2512.

30. LeRoith D. Insulin-like growth factor I receptor signaling--overlapping or redundant pathways? Endocrinology. 2000;141(4):1287-1288.

31. Dietrich P, Dragatsis I, Xuan S, Zeitlin S, Efstratiadis A. Conditional mutagenesis in mice with heat shock promoter-driven cre transgenes. Mamm Genome. 2000;11(3):196-205.

32. Mohan S. Insulin-like growth factor binding proteins in bone cell regulation. Growth Regul. 1993;3(1):67-70.

33. Rajpathak SN, et al. The role of insulin-like growth factor-I and its binding proteins in glucose homeostasis and type 2 diabetes. Diabetes Metab Res Rev. 2009;25(1):3-12.

34. Laviola L, Natalicchio A, Giorgino F. The IGF-I signaling pathway. Curr Pharm Des. 2007;13(7):663-669.

35. Kim H, Barton E, Muja N, Yakar S, Pennisi P, LeRoith D. Intact insulin and insulin-like growth factor-I receptor signaling is required for growth hormone effects on skeletal muscle growth and function in vivo. Endocrinology. 2005;146(4):1772-1779.

36. Rando TA, Blau HM. Primary mouse myoblast purification, characterization, and transplantation for cell-mediated gene therapy. J Cell Biol. 1994;125(6):1275-1287.

37. Urban RJ, et al. Testosterone administration to elderly men increases skeletal muscle strength and protein synthesis. Am J Physiol. 1995;269(5 pt 1):E820-E826.

38. Gentile MA, et al. Androgen-mediated improvement of body composition and muscle function involves a novel early transcriptional program including IGF1, mechano growth factor, and induction of \{beta\}-catenin. J Mol Endocrinol. 2010; 44(1):55-73.

39. Tremblay F, et al. Identification of IRS-1 Ser-1101 as a target of S6K1 in nutrient- and obesity-induced insulin resistance. Proc Natl Acad Sci U S A. 2007; 104(35):14056-14061.

40. Himpe E, Kooijman R. Insulin-like growth factor-I receptor signal transduction and the Janus Kinase/ Signal Transducer and Activator of Transcription (JAK-STAT) pathway. Biofactors. 2009;35(1):76-81.

41. Cuneo RC, Salomon F, Wiles CM, Sonksen PH. Skeletal muscle performance in adults with growth hormone deficiency. Horm Res. 1990;33 suppl 4:55-60.

42. Cuneo RC, Salomon F, Wiles CM, Hesp R, Sonksen $\mathrm{PH}$. Growth hormone treatment in growth hormone-deficient adults. I. Effects on muscle mass and strength. J Appl Physiol. 1991;70(2):688-694.

43. Mauras N, Attie KM, Reiter EO, Saenger P, Baptista J. High dose recombinant human growth hormone $(\mathrm{GH})$ treatment of GH-deficient patients in puberty increases near-final height: a randomized, multicenter trial. Genentech, Inc., Cooperative Study Group. J Clin Endocrinol Metab. 2000; 85(10):3653-3660.

44. Mauras N, et al. Insulin-like growth factor I and growth hormone $(\mathrm{GH})$ treatment in $\mathrm{GH}$-deficient humans: differential effects on protein, glucose, lipid, and calcium metabolism. J Clin Endocrinol Metab. 2000;85(4):1686-1694.

45. Mauras N. Growth hormone therapy in the glucocorticosteroid-dependent child: metabolic and linear growth effects. Horm Res. 2001;56 suppl 1:13-18.

46. Cotes PM, Reid E, Young FG. Diabetogenic action of pure anterior pituitary growth hormone. Nature. 1949;164(4162):209-211.

47. Houssay BA, Rodriguez RR. Diabetogenic action of different preparations of growth hormone. Endocrinology. 1953;53(1):114-116.

48. Rommel C, et al. Mediation of IGF-1-induced skeletal myotube hypertrophy by $\mathrm{PI}(3) \mathrm{K} / \mathrm{Akt} / \mathrm{mTOR}$ and PI(3)K/Akt/GSK3 pathways. Nat Cell Biol. 2001;3(11):1009-1013.

49. Liu JP, Baker J, Perkins AS, Robertson EJ, Efstratiadis A. Mice carrying null mutations of the genes encoding insulin-like growth factor I (Igf-1) and type 1 IGF receptor (Igf1r). Cell. 1993;75(1):59-72.

50. Daugaard JR, et al. Effect of 6 months of GH treatment on myosin heavy chain composition in GH-deficient patients. Eur J Endocrinol. 1999; 141(4):342-349.

51. Lovett FA, et al. Convergence of Igf2 expression and adhesion signalling via RhoA and p38 MAPK enhances myogenic differentiation. J Cell Sci. 2006; 119(pt 23):4828-4840.

52. Ren H, Yin P, Duan C. IGFBP-5 regulates muscle cell differentiation by binding to IGF-II and switching on the IGF-II auto-regulation loop. J Cell Biol. 2008; 182(5):979-991.

53. Bratusch-Marrain PR, Smith D, DeFronzo RA. The effect of growth hormone on glucose metabolism and insulin secretion in man. J Clin Endocrinol Metab. 1982;55(5):973-982.

54. Davidson MB, Shen DC, Venkatesan N, Sladen G. In vivo insulin antagonism but evanescent in vitro tissue effect in rats with growth hormone-secreting tumors. J Endocrinol Invest. 1987;10(6):569-574.

55. Rizza RA, Mandarino LJ, Gerich JE. Effects of growth 
hormone on insulin action in man. Mechanisms of insulin resistance, impaired suppression of glucose production, and impaired stimulation of glucose utilization. Diabetes. 1982;31(8 pt 1):663-669.

56. Hopwood NJ, Forsman PJ, Kenny FM, Drash AL. Hypoglycemia in hypopituitary children. Am J Dis Child. 1975;129(8):918-926.

57. Bougneres PF, Artavia-Loria E, Ferre P, Chaussain $\mathrm{JL}$, Job JC. Effects of hypopituitarism and growth hormone replacement therapy on the production and utilization of glucose in childhood. J Clin Endocrinol Metab. 1985;61(6):1152-1157.

58. Daugaard JR, Laustsen JL, Hansen BS, Richter EA. Insulin action in growth hormone-deficient and age-matched control rats: effect of growth hormone treatment. J Endocrinol. 1999;160(1):127-135.

59. Bonkowski MS, Rocha JS, Masternak MM, Al Regaiey KA, Bartke A. Targeted disruption of growth hormone receptor interferes with the beneficial actions of calorie restriction. Proc Natl Acad Sci US A. 2006;103(20):7901-7905.

60. Coschigano KT, Holland AN, Riders ME, List EO, Flyvbjerg A, Kopchick JJ. Deletion, but not antagonism, of the mouse growth hormone receptor results in severely decreased body weights, insulin, and insulin-like growth factor I levels and increased life span. Endocrinology. 2003;144(9):3799-3810.

61. Panici JA, et al. Is altered expression of hepatic insulin-related genes in growth hormone receptor knockout mice due to $\mathrm{GH}$ resistance or a difference in biological life spans? J Gerontol A Biol Sci Med Sci. 2009;64(11):1126-1133.

62. Kim CH, et al. MKR mice are resistant to the metabolic actions of both insulin and adiponectin: discordance between insulin resistance and adiponectin responsiveness. Am J Physiol Endocrinol Metab. 2006;291(2):E298-E305.

63. Hosui A, Hennighausen L. Genomic dissection of the cytokine-controlled STAT5 signaling network in liver. Physiol Genomics. 2008;34(2):135-143.

64. Klover P, Hennighausen L. Postnatal body growth is dependent on the transcription factors signal transducers and activators of transcription $5 \mathrm{a} / \mathrm{b}$ in muscle: a role for autocrine/paracrine insulin-like growth factor I. Endocrinology. 2007;148(4):1489-1497.

65. Klover P, Chen W, Zhu BM, Hennighausen L. Skeletal muscle growth and fiber composition in mice are regulated through the transcription factors STAT $5 \mathrm{a} / \mathrm{b}$ : linking growth hormone to the androgen receptor. FASEB J. 2009;23(9):3140-3148.

66. Ozes ON, et al. A phosphatidylinositol 3-kinase/ Akt/mTOR pathway mediates and PTEN antagonizes tumor necrosis factor inhibition of insulin signaling through insulin receptor substrate-1. Proc Natl Acad Sci U S A. 2001;98(8):4640-4645.

67. De Fea K, Roth RA. Protein kinase C modulation of insulin receptor substrate-1 tyrosine phos phorylation requires serine 612. Biochemistry. 1997;
36(42):12939-12947

68. Ma L, Chen Z, Erdjument-Bromage H, Tempst $P$, Pandolfi PP. Phosphorylation and functional inactivation of TSC 2 by Erk implications for tuberous sclerosis and cancer pathogenesis. Cell. 2005;121(2):179-193.

69. Buller CL, et al. A GSK-3/TSC2/mTOR pathway regulates glucose uptake and GLUT1 glucose transporter expression. Am J Physiol Cell Physiol. 2008;295(3):C836-843.

70. Hayashi AA, Proud CG. The rapid activation of protein synthesis by growth hormone requires signaling through mTOR. Am J Physiol Endocrinol Metab. 2007;292(6):E1647-1655.

71. Zhang Y, et al. Growth hormone (GH)-induced dimerization inhibits phorbol ester-stimulated GH receptor proteolysis. J Biol Chem. 2001;276(27):24565-24573.

72. Fan Y, et al. Liver-specific deletion of the growth hormone receptor reveals essential role of growth hormone signaling in hepatic lipid metabolism. J Biol Chem. 2009;284(30):19937-19944.

73. Fulzele K, DiGirolamo DJ, Liu Z, Xu J, Messina JL, Clemens TL. Disruption of the insulin-like growth factor type 1 receptor in osteoblasts enhances insulin signaling and action. J Biol Chem. 2007;282(35):25649-25658.

74. Nagy A. Cre recombinase: the universal reagent for genome tailoring. Genesis. 2000;26(2):99-109. 